Supporting information

\title{
Mono-Cadmium vs Mono-Mercury Doping of Au25 nanoclusters
}

Chuanhao Yao ${ }^{\dagger}{ }^{\S}$ Yue-jian Lin, ${ }^{\star, \S}$ Jinyun Yuan,,${ }^{\S}$ Lingwen Liao, ${ }^{\dagger}$ Min Zhu, ${ }^{\dagger}$ Lin-hong Weng, ${ }^{*}$, Jinlong Yang, ", and Zhikun $\mathrm{Wu}^{*}, \dagger$

${ }^{\dagger}$ Institute of Solid State Physics, Chinese Academy of Sciences, Hefei, Anhui 230031, China. Email: zkwu@issp.ac.cn

${ }^{\ddagger}$ Shanghai Key Laboratory of Molecular Catalysis and Innovative Material, Department of Chemistry, Fudan University, Shanghai 200433, China. Email: lhweng@fudan.edu.cn

"Hefei National Laboratory for Physics Sciences at the Microscale, University of Science and Technology of China, Hefei, Anhui230026, China. Email: jlyang@ustc.edu.cn

\section{Experimental Methods}

Chemicals. All chemicals are commercially available and used as received. The water used in all experiments was ultrapure (resistivity $18.2 \mathrm{M} \Omega \mathrm{cm}$ ), produced with a Milli-Q NANO pure water system. Tetraoctylammonium bromide (TOABr, 98.0\%), was obtained from Aladdin. 2-Phenylethanethiol $\left(\mathrm{PhC}_{2} \mathrm{H}_{4} \mathrm{SH}, 99.0 \%\right)$, Sodium borohydride $\left(\mathrm{NaBH}_{4}, \quad 99.0 \%\right)$ were purchased from Sigma-Aldrich. Tetrachloroauric(III) acid $\left(\mathrm{HAuCl}_{4} \cdot 4 \mathrm{H}_{2} \mathrm{O}, \quad 99.7 \%\right), \quad \mathrm{Zn}\left(\mathrm{NO}_{3}\right)_{2} \cdot 6 \mathrm{H}_{2} \mathrm{O} \quad(99.5 \%)$, $\mathrm{Co}\left(\mathrm{NO}_{3}\right)_{2} \cdot 6 \mathrm{H}_{2} \mathrm{O} \quad(99.5 \%), \quad \mathrm{Ni}\left(\mathrm{NO}_{3}\right)_{2} \cdot 6 \mathrm{H}_{2} \mathrm{O} \quad(99.5 \%), \quad \mathrm{Cd}\left(\mathrm{NO}_{3}\right)_{2} \cdot 4 \mathrm{H}_{2} \mathrm{O} \quad(99.5 \%)$, tetrahydrofuran (THF, 99.0\%), acetonitrile $\left(\mathrm{CH}_{3} \mathrm{CN}\right.$, 99.0\%), methanol (99.5\%), Dichloromethane (AR), Petroleum ether (AR) and toluene (99.5\%) were purchased from Sinopharm chemical reagent co., ltd.

Synthesis of anion $\mathrm{Au}_{25}(\mathrm{PET})_{18}$ was referred to our previous work. ${ }^{1}$

Reaction of active metal ions with $\mathbf{A u}_{25}(\text { PET })_{18} .31 .5 \mathrm{mg}(0.004 \mathrm{~m} \mathrm{~mol})$ of anion

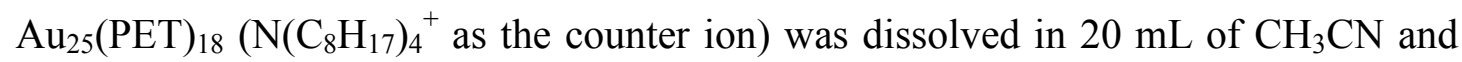
the resultant solution was divided equally into 4 parts. Freshly prepared acetonitrile solution containing 20 equivalents of $\mathrm{Cd}\left(\mathrm{NO}_{3}\right)_{2}, \mathrm{Co}\left(\mathrm{NO}_{3}\right)_{2}$ or $\left.\mathrm{Ni}\left(\mathrm{NO}_{3}\right)_{2}\right)$ was added to every part under vigorous stirring. The reaction was carried out at $60{ }^{\circ} \mathrm{C}$ in a water bath. Over half an hour, the solution became almost clear and a large amount of black solid precipitated out. The collected solid was washed with excess $\mathrm{CH}_{3} \mathrm{CN}$ and dried 
in a vacuum for further characterization. The reaction between $\mathrm{Zn}\left(\mathrm{NO}_{3}\right)_{2}$ and anion $\mathrm{Au}_{25}(\mathrm{PET})_{18}$ was referred to the above protocols. However, the product cannot precipitate out from acetonitrile solution. The product was collected by rotary evaporation and subsequently washed three times with excess water to remove the free ions.

Recrystallization of $\mathbf{A u}_{24} \mathbf{C d}(\mathbf{P E T})_{18}$. Recrystallization of $\mathrm{Au}_{24} \mathrm{Cd}(\mathrm{PET})_{18}$ was conducted in a mixture of toluene and methanol. Briefly, $15 \mathrm{mg}$ of $\mathrm{Au}_{24} \mathrm{Cd}(\mathrm{PET})_{18}$ was dissolved in toluene/methanol $(5 / 1, \mathrm{~V} / \mathrm{V})$, followed by slow vapor $\left(\mathrm{CH}_{3} \mathrm{OH}\right)$ diffusion into the cluster solution. Over 6 days at room temperature, black crystals with a regular geometrical shape were observed. The obtained $\mathrm{Au}_{24} \mathrm{Cd}(\mathrm{PET})_{18}$ crystals were analyzed by X-ray crystallography. The yield of $\mathrm{Au}_{24} \mathrm{Cd}(\mathrm{PET})_{18}$ is $56 \%$.

Transformation of $\mathbf{A u}_{24} \mathbf{C d}(\mathbf{P E T})_{18}$ to $\mathbf{A u}_{24} \mathbf{H g}(\mathbf{P E T})_{18} \cdot 29.3 \mathrm{mg}(0.004 \mathrm{mmol})$ of $\mathrm{Au}_{24} \mathrm{Cd}(\mathrm{PET})_{18}$ was dissolved in $3 \mathrm{~mL}$ of THF. Subsequently, a freshly prepared aqueous solution containing 1 equivalent of $\mathrm{Hg}\left(\mathrm{NO}_{3}\right)_{2}$ was added under vigorous stirring. The reaction was accomplished immediately, and a black solid precipitated out after the addition of excess methanol. The solid product was washed three times with methanol and water to remove the free ions. Crystallization was performed by the diffusion of methanol into a toluene solution of the nanoclusters. After a week, flower-like dark crystals of $\mathrm{Au}_{24} \mathrm{Hg}(\mathrm{PET})_{18}$ were observed. The yield of $\mathrm{Au}_{24} \mathrm{Hg}(\mathrm{PET})_{18}$ was $76 \%$.

Characterizations. Matrix-assisted laser desorption ionization time of flight mass spectrometry (MALDI-TOF-MS) was performed on an Autoflex Speed TOF/TOF mass spectrometer (Bruker) in positive mode and Compass IsotopePattern was used to simulate the isotopic pattern. Trans-2-[3-(4-tert-butylphenyl)-2-methyl-2-propenylidene] malononitrile (DCTB) was used as the matrix, and the molar ratio of cluster to matrix was 1:1000. The UV/vis/NIR absorption spectra were measured on a UV-2600 spectrophotometer at room temperature. Thermal gravimetric analysis (TGA) ( $\sim 2 \mathrm{mg}$ sample used) was conducted in a $\mathrm{N}_{2}$ atmosphere (flow rate $\sim 50$ $\mathrm{mL} / \mathrm{min}$ ) on a TG/DTA 6300 analyzer at a heating rate of $10^{\circ} \mathrm{C} / \mathrm{min}$. X-ray intensity data were collected at 123 and $275 \mathrm{~K}$ using graphite-monochromated Mo-Ka radiation $(0.71073 \AA)$ with a Bruker Smart Apex II CCD diffractometer. Data reduction included absorption corrections by the multi-scan method using SADABS. The 
structure was solved by direct methods and refined by full matrix least squares using the SHELXTL 6.1 bundled software package.

Theoretical method. All calculations were carried out using Density Functional Theory (DFT) with the pure functional $\mathrm{PBE}^{2,3}$ and all electron basis set 6-31g (d, p) for $\mathrm{H}, \mathrm{C}$, and S, pseudopotential basis set LANL2DZ for Au, as implemented in the Gaussian 09 program package. ${ }^{4}$ The PBE functional has been verified to be reliable for gold cluster systems. ${ }^{5,6}$ In order to confirm the relative stability of the three isomers of $\mathrm{Au}_{24} \mathrm{Cd}(\mathrm{PET})_{18}$, their single point energies were calculated. These three isomers were constructed by substituting a $\mathrm{Au}$ with a $\mathrm{Cd}$ in the core, inner-shell, and outer-shell of $\mathrm{Au}_{25}$, respectively.

Time-dependent density functional calculations $\left(\right.$ TDDFT) ${ }^{7}$ were performed to reproduce the experimental UV/vis/NIR spectrum. Since the tail group of the thiolate has no influence to the optical absorption of $\mathrm{Au}_{38}(\mathrm{SR})_{24}$ clusters, ${ }^{8}$ we use $-\mathrm{CH}_{3}$ to replace $-\mathrm{R}$ group for minimizing computational costs. The Gaussian half-width at half-height of $0.15 \mathrm{eV}$ in the Multiwfn software ${ }^{9}$ was used to simulate the UV/vis/NIR spectrum. 


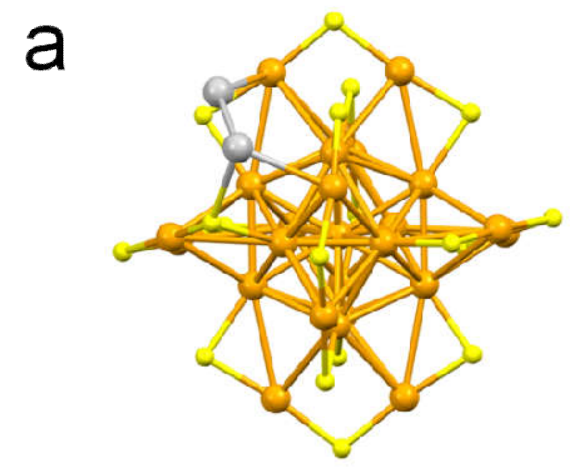

$\operatorname{Au}_{25} \mathrm{Ag}_{2}(\mathrm{PET}){ }_{18}$

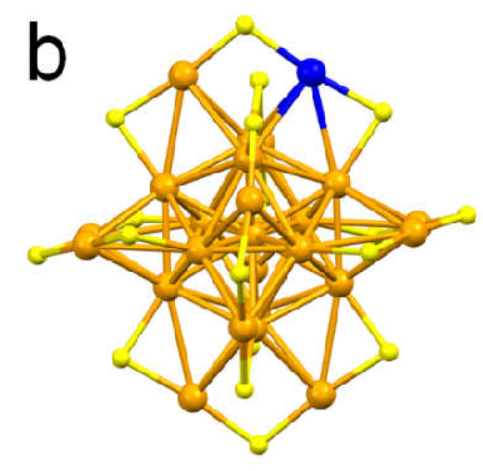

$\mathrm{Au}_{24 \mathrm{Hg}}(\mathrm{PET}){ }_{18}$

Figure S1 Structure of $\mathrm{Au}_{25} \mathrm{Ag}_{2}(\mathrm{PET})_{18}$ (a) and $\mathrm{Au}_{24} \mathrm{Hg}(\mathrm{PET})_{18}$ (b).(Color labels: $\mathrm{Au}$, orange; Ag, grey; $\mathrm{Hg}$, blue; $\mathrm{S}$, yellow. $\mathrm{C}$ and $\mathrm{H}$ atoms were omitted for clarity).

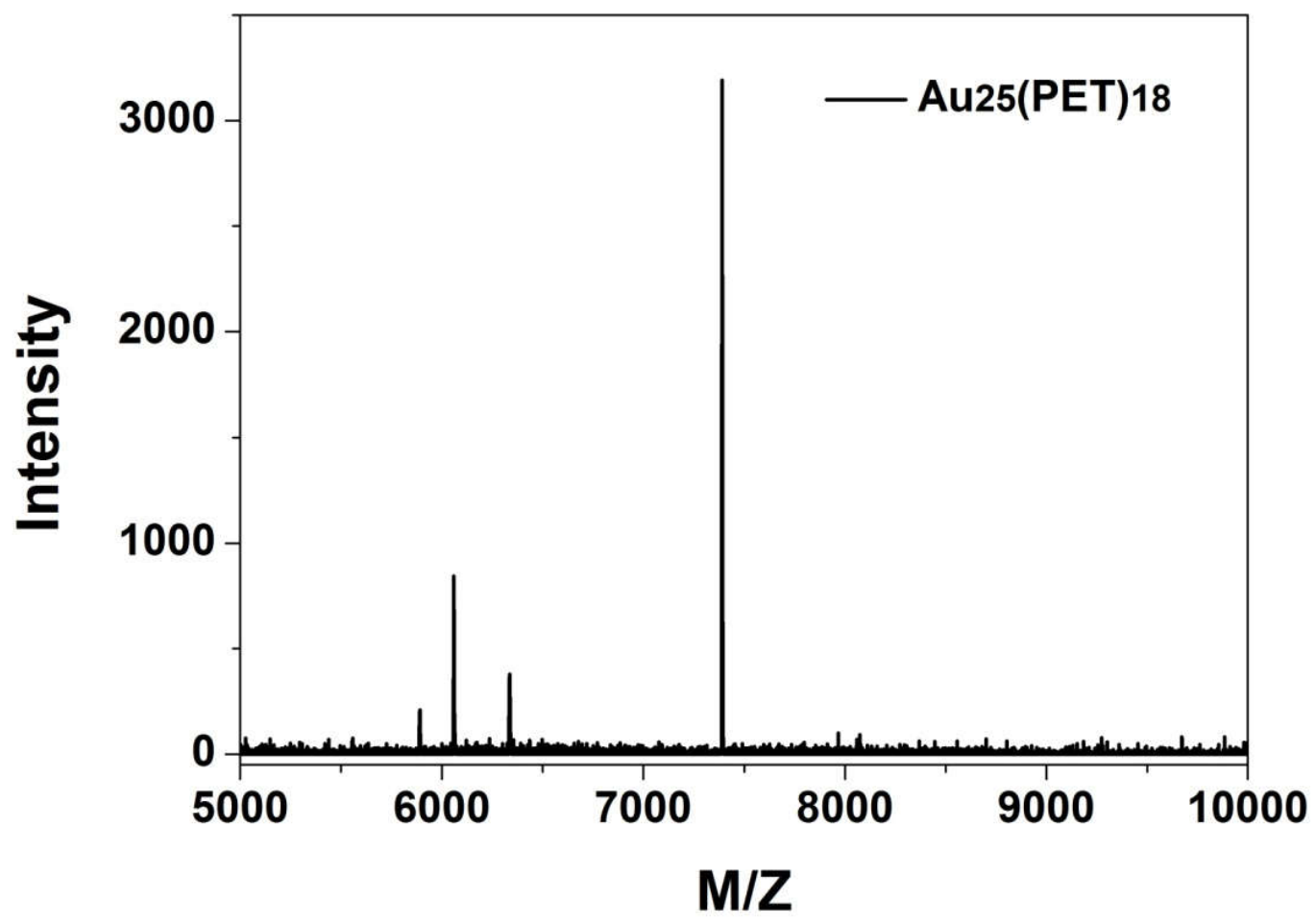

Figure S2 MALDI-TOF-MS spectrum of $\left[\mathrm{Au}_{25}(\mathrm{PET})_{18}\right]^{-}$(acquired in negative mode). 


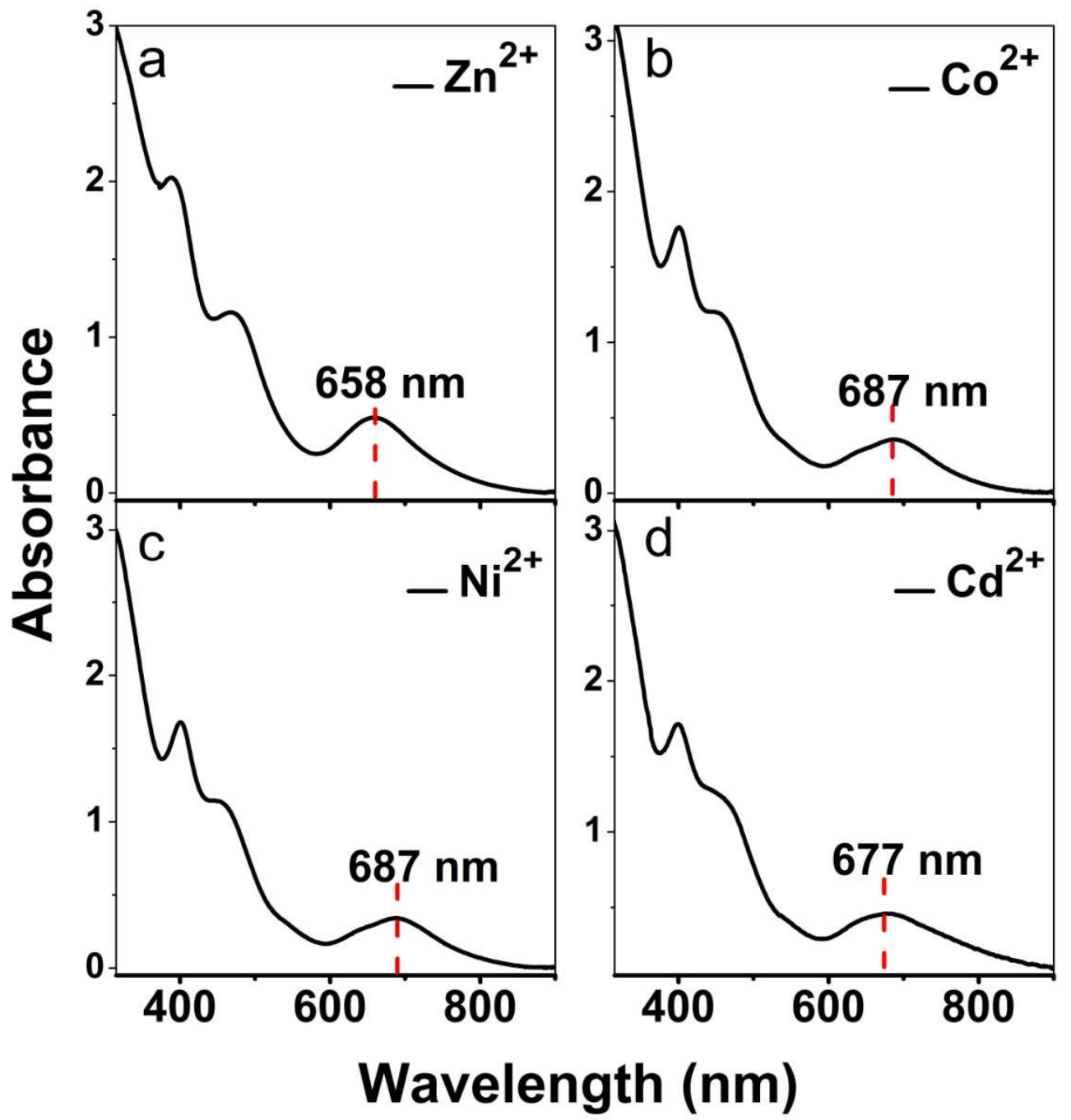

Figure S3 UV/vis/NIR absorption spectra of the products obtained from the reaction between $\left[\mathrm{Au}_{25}(\mathrm{PET})_{18}\right]^{-}$and $\mathrm{Zn}^{2+}(\mathrm{a}), \mathrm{Co}^{2+}(\mathrm{b}), \mathrm{Ni}^{2+}(\mathrm{c})$ or $\mathrm{Cd}^{2+}(\mathrm{d})$, respectively. 


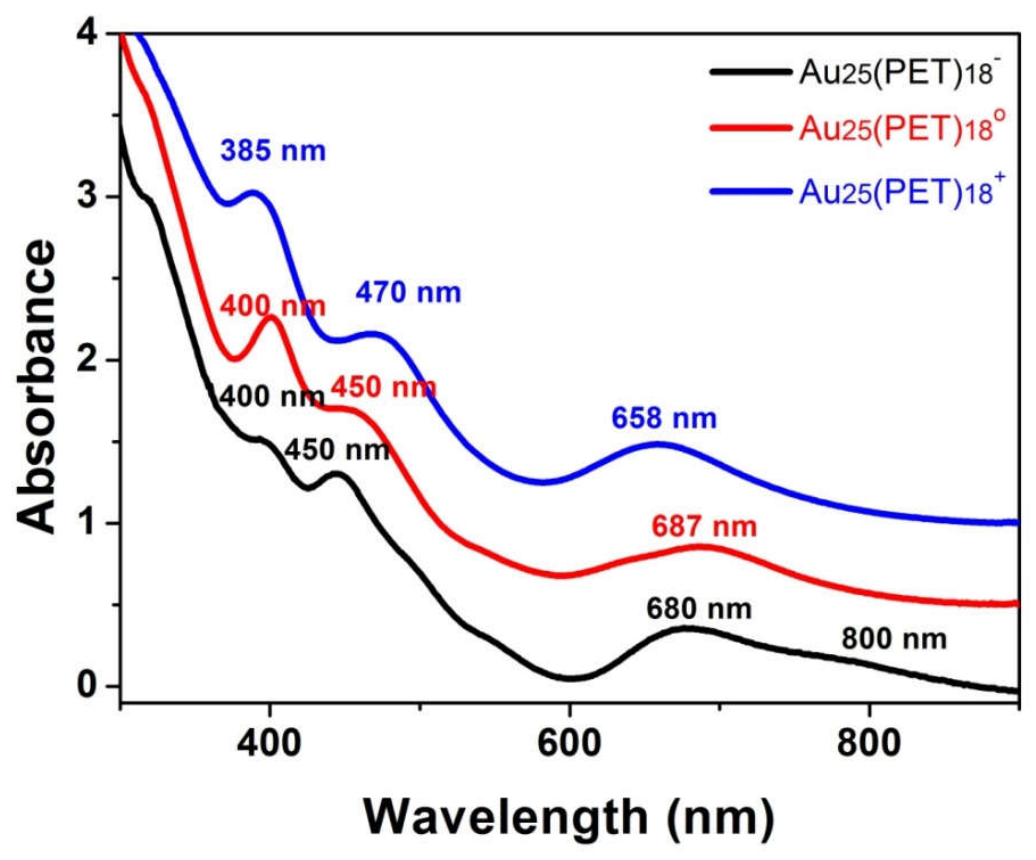

Figure $\mathbf{S 4}$ UV/vis/NIR absorption spectra of variously charged $\left[\mathrm{Au}_{25}(\mathrm{PET})_{18}\right]^{\mathrm{q}}(\mathrm{q}=-1$, $0,+1)$.

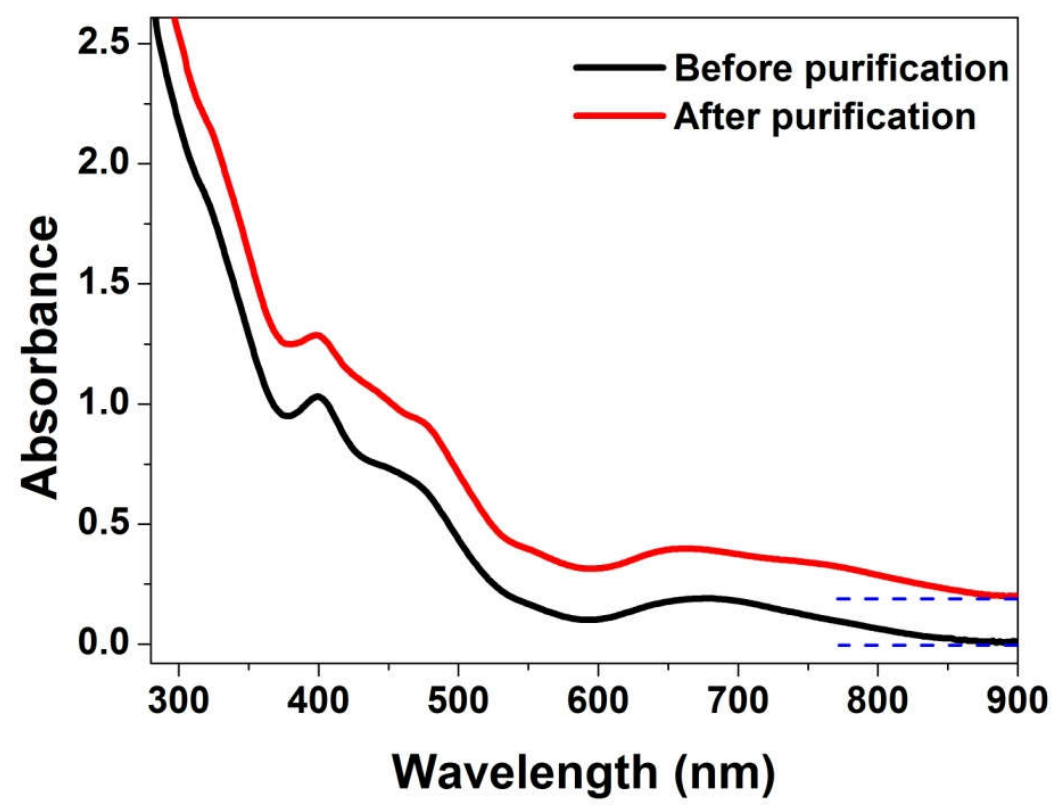

Figure S5 UV/vis/NIR absorption spectra of $\mathrm{Au}_{24} \mathrm{Cd}(\mathrm{PET})_{18}$ before and after purification. 


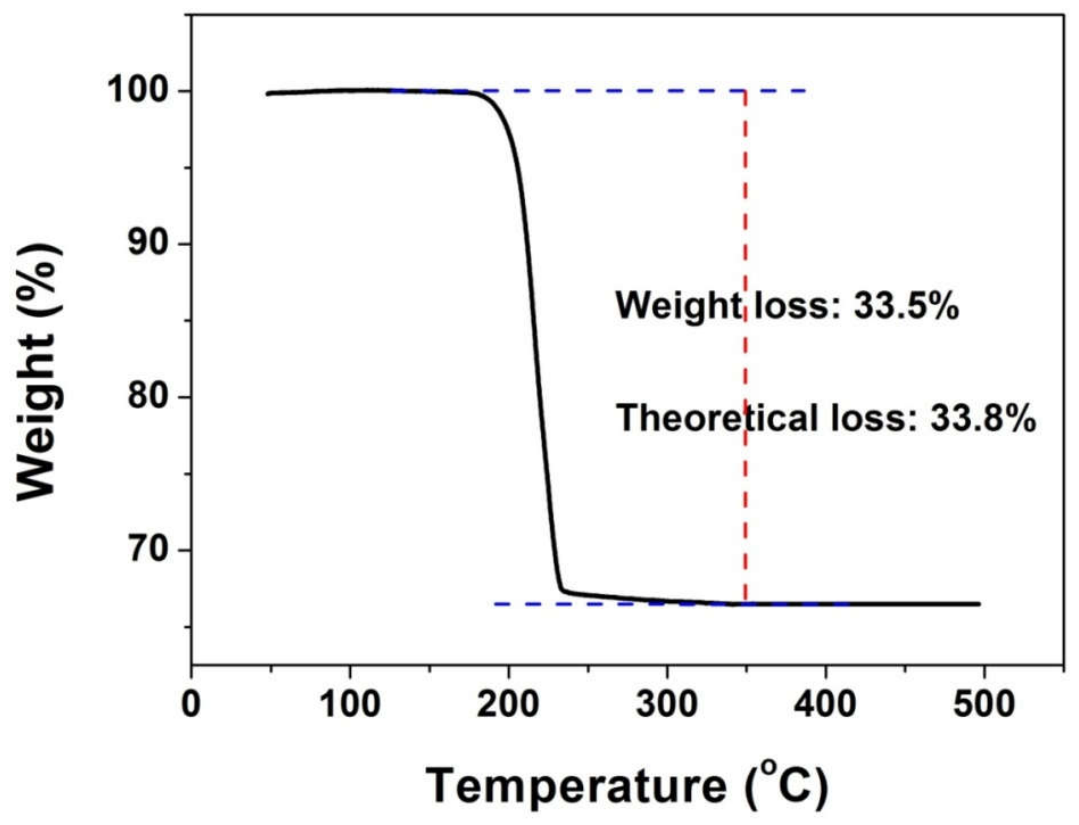

Figure S6 Thermogravimetric analysis (TGA) of $\mathrm{Au}_{24} \mathrm{Cd}(\mathrm{PET})_{18}$.

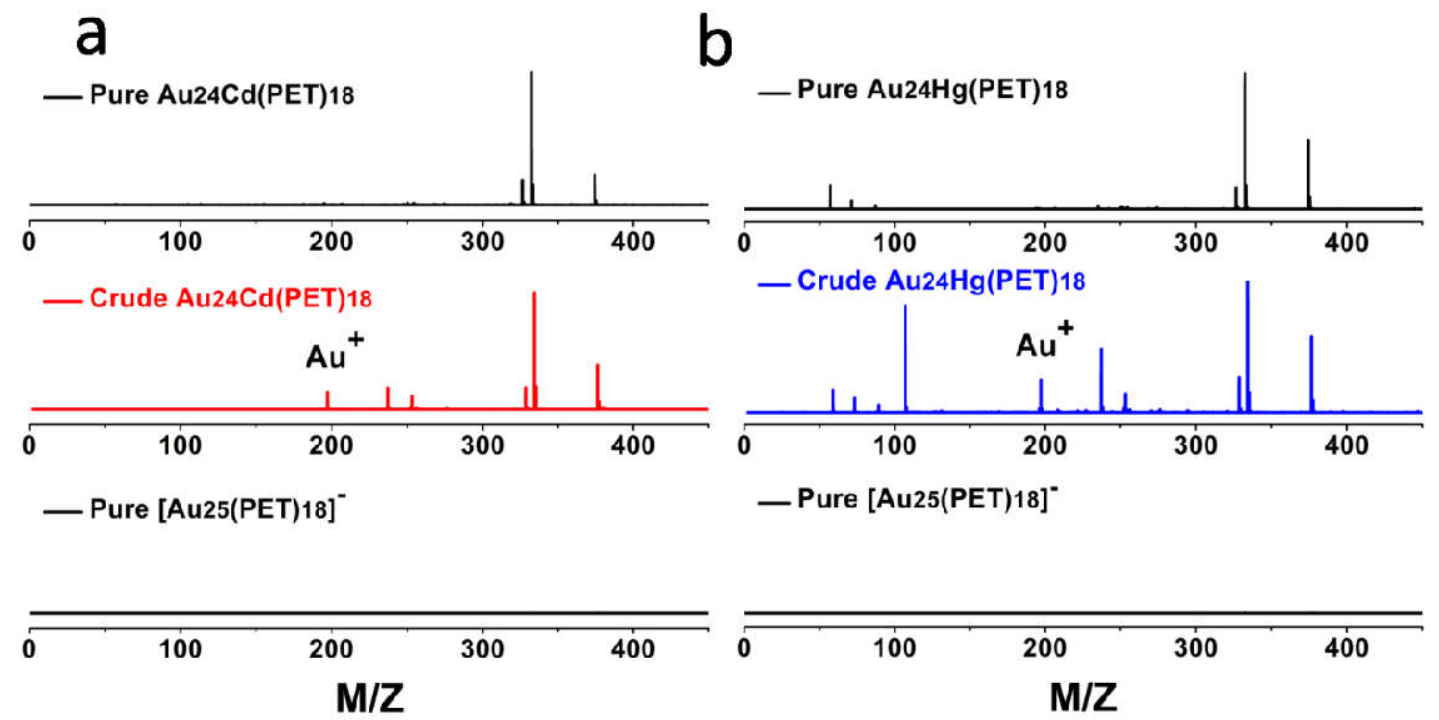

Figure S7 Detected $\mathrm{Au}^{+}$in the crude products of the reactions between $\left[\mathrm{Au}_{25}(\mathrm{PET})_{18}\right]^{-}$ and $\mathrm{Cd}^{2+}(\mathrm{a})$, or $\mathrm{Hg}^{2+}$ (b) by MALDI-TOF-MS (acquired in positive ionization mode). 


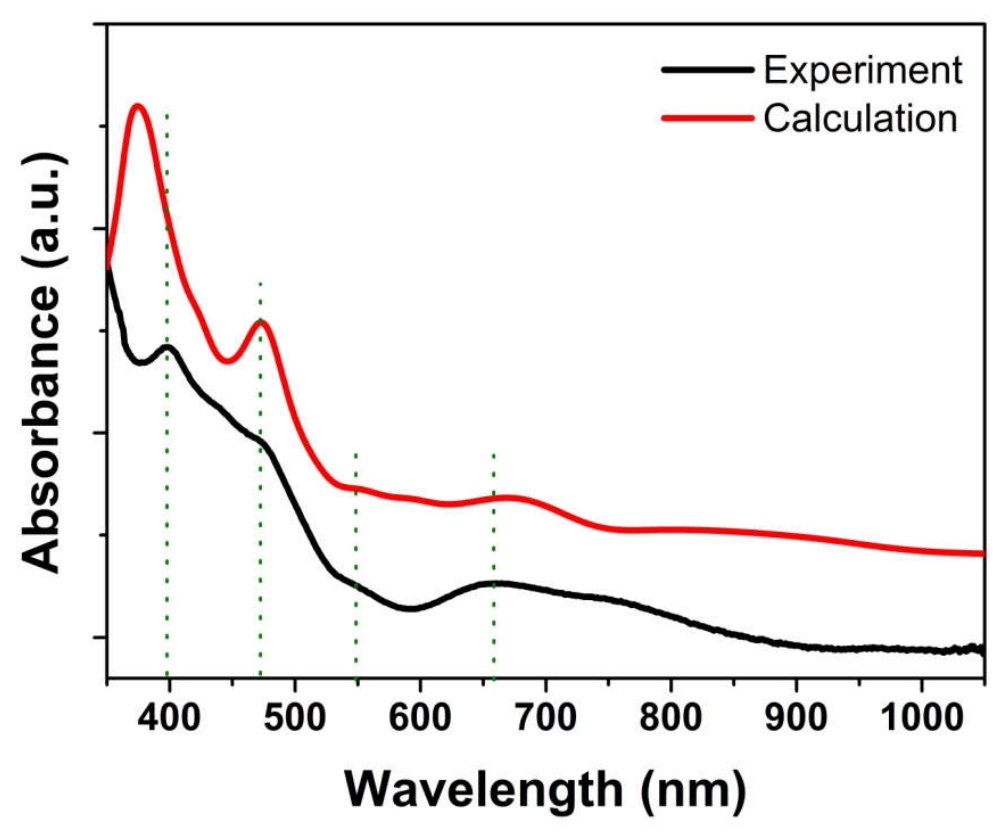

Figure S8 Experimental and TDDFT simulated UV/vis/NIR adsorption spectra of $\mathrm{Au}_{24} \mathrm{Cd}(\mathrm{PET})_{18}$.

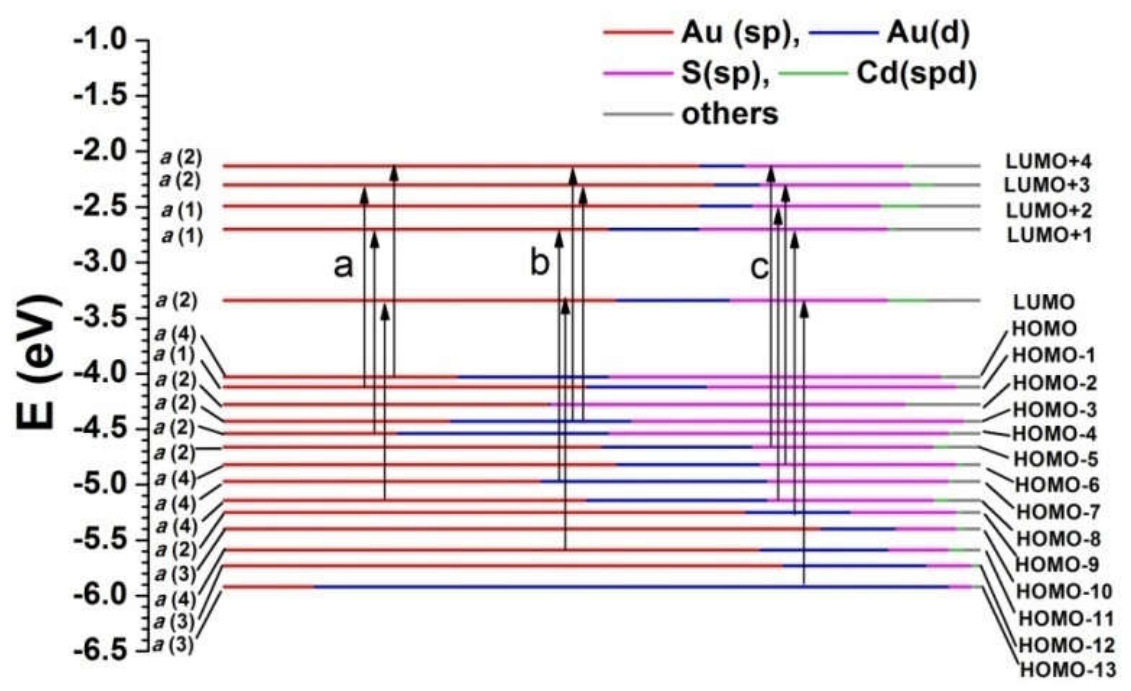

Figure S9 Kohn-Sham orbital energy level diagram for a model compound $\mathrm{Au}_{24} \mathrm{Cd}\left(\mathrm{SCH}_{3}\right)_{18}$. The energies are in units of eV. Each $\mathrm{KS}$ orbital is drawn to indicate the relative contributions (line length with color labels) of the atomic orbitals of $\mathrm{Au}$ (6sp) in red, $\mathrm{Au}(5 \mathrm{~d})$ in blue, $\mathrm{S}(3 \mathrm{sp})$ in purple, $\mathrm{Cd}(\mathrm{spd})$ in green and others in gray. The left column of the KS orbitals shows the orbitals symmetry and degeneracy (in parenthesis) (Orbitals with energy difference less than $\sim 0.1 \mathrm{eV}$ was degenerated); the right column shows the HOMO and LUMO sets. 


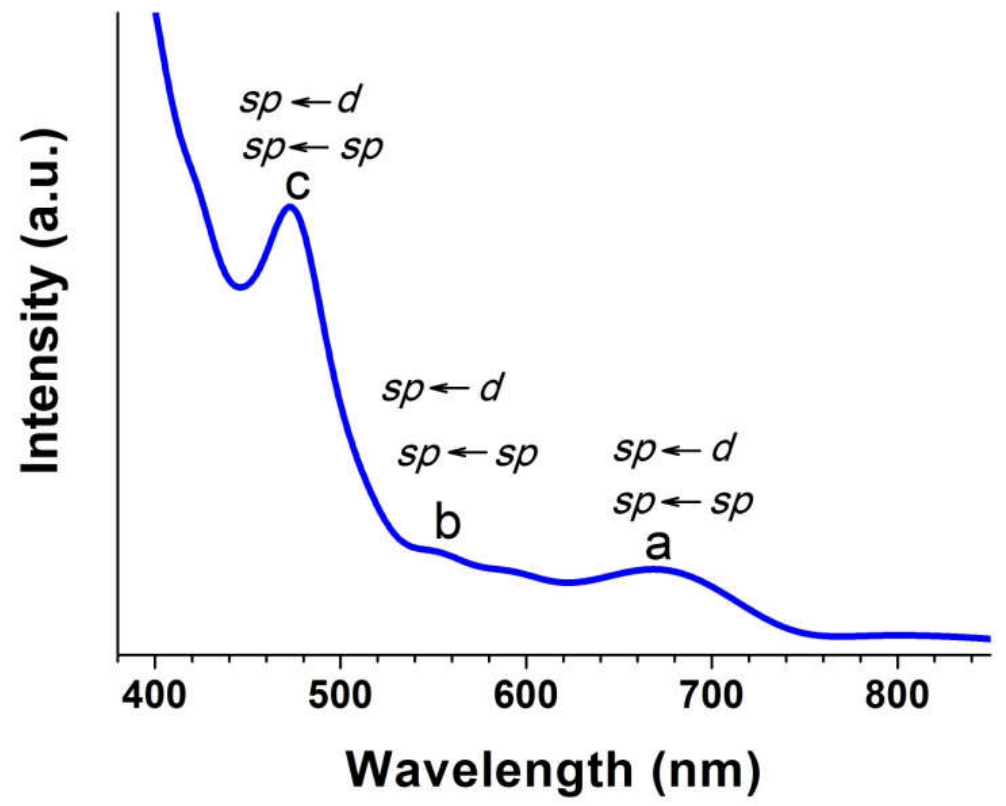

Figure S10 Calculated absorption spectrum of $\mathrm{Au}_{24} \mathrm{Cd}\left(\mathrm{SCH}_{3}\right)_{18}$.

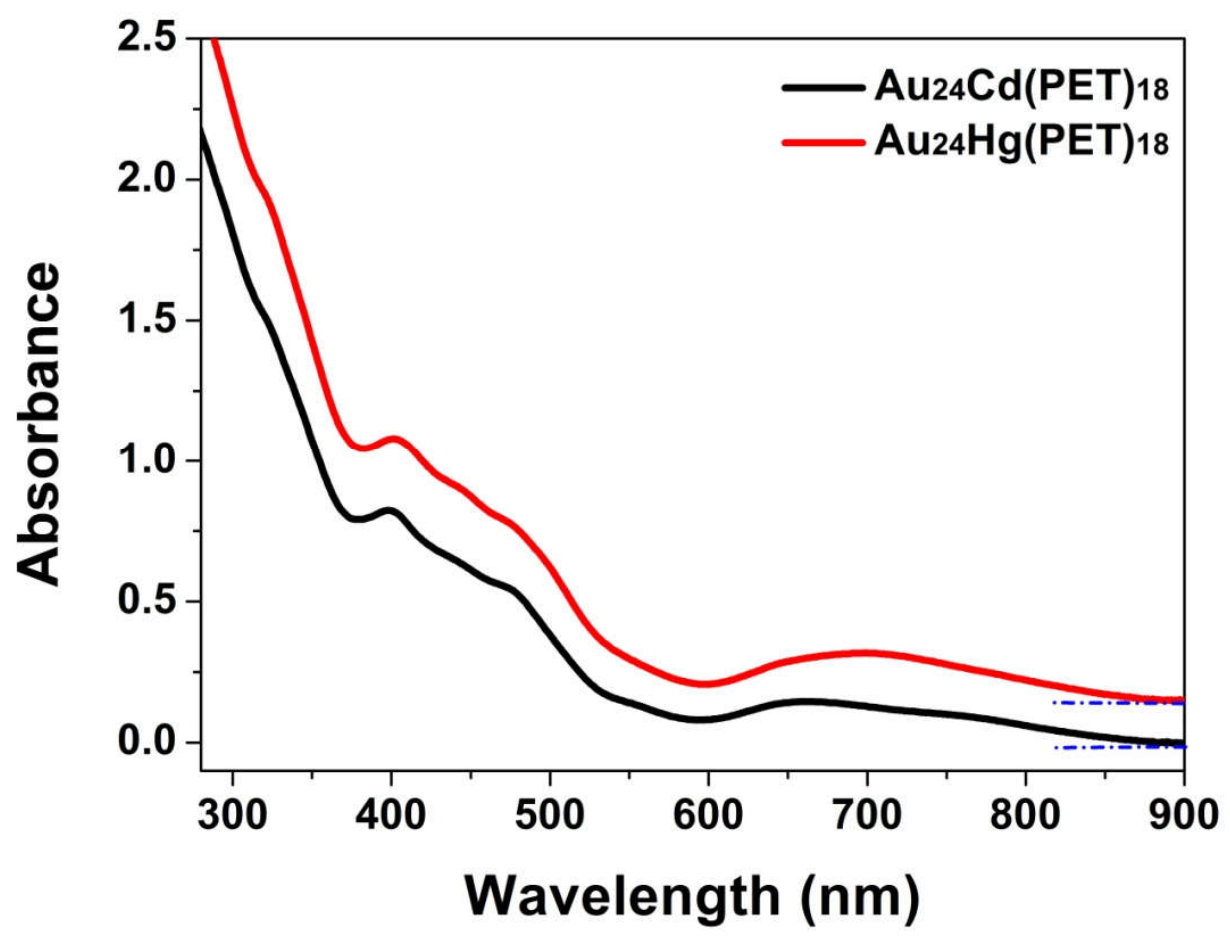

Figure S11 UV/vis/NIR absorption spectra comparison between $\mathrm{Au}_{24} \mathrm{Cd}(\mathrm{PET})_{18}$ and $\mathrm{Au}_{24} \mathrm{Hg}(\mathrm{PET})_{18}$. 


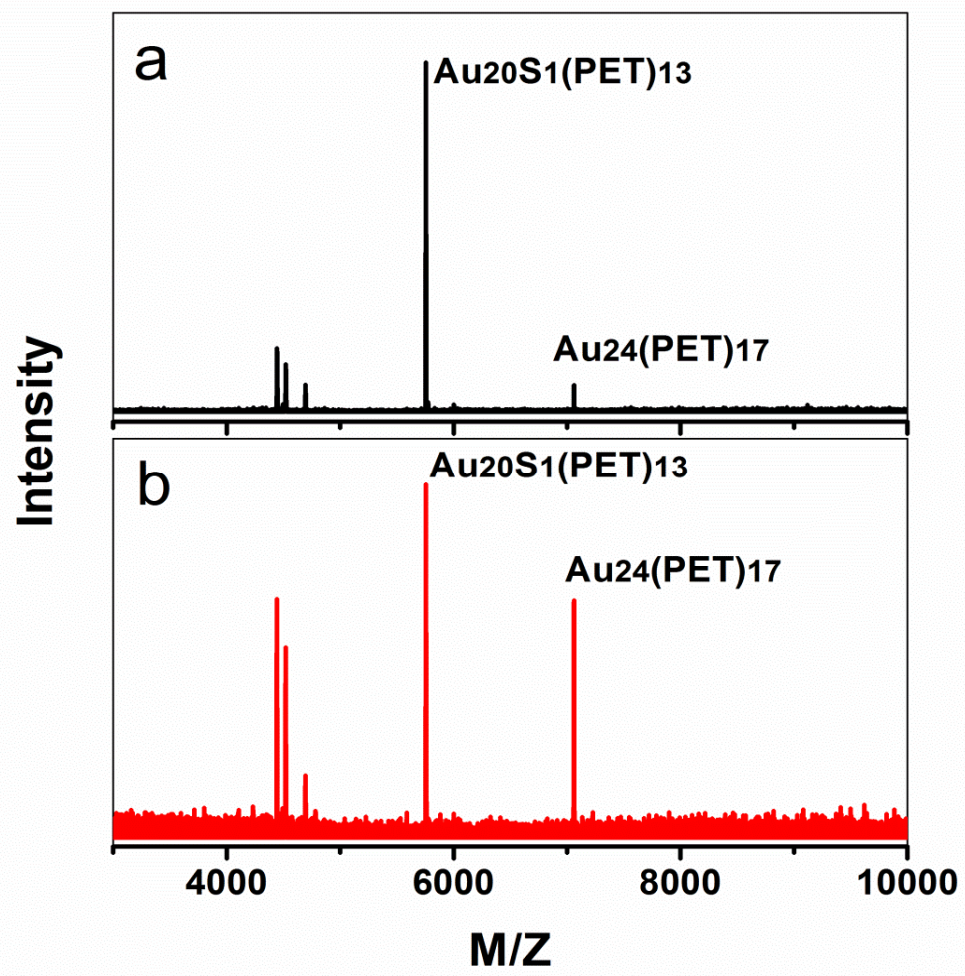

Figure S12 MALDI-TOF-MS spectra of $\mathrm{Au}_{24} \mathrm{Hg}(\mathrm{PET})_{18}$ obtained via our previous method $^{10}$ (a) or the current transferring from $\mathrm{Au}_{24} \mathrm{Cd}(\mathrm{PET})_{18}$ (b).

\section{References}

1 Wu, Z.; Suhan, J.; Jin, R. J. Mater. Chem., 2009, 19, 622.

2 Perdew, J. P., Burke, K., Ernzerhof, M., Phys. Rev. Lett., 1996, 77, 3865.

3 Perdew, J. P., Burke, K., Ernzerhof, M., Phys. Rev. Lett., 1997, 78, 1396.

4 Frisch, M. J.; Trucks, G. W.; Schlegel, H. B.; Scuseria, G. E.; Robb, M. A.; Cheeseman, J. R.; Scalmani, G.; Barone, V.; Mennucci, B.; Petersson, G. A.; Nakatsuji, H.; Caricato, M.; Li, X.; Hratchian, H. P.; Izmaylov, A. F.; Bloino, J.; Zheng, G.; Sonnenberg, J. L.; Hada, M.; Ehara, M.; Toyota, K.; Fukuda, R.;

Hasegawa, J.; Ishida, M.; Nakajima, T.; Honda, Y.; Kitao, O.; Nakai, H.; Vreven, T.; Montgomery, Jr., J. A.; Peralta, J. E.; Ogliaro, F.; Bearpark, M.; Heyd, J. J.; Brothers, E.; Kudin, K. N.; Staroverov, V. N.; Keith, T.; Kobayashi, R.; Normand, J.; Raghavachari, K.; Rendell, A.; Burant, J. C.; Iyengar, S. S.; Tomasi, J.; Cossi,M.; Rega, N.; Millam, J. M.; Klene, M.; Knox, J. E.; Cross, J. B.; Bakken, V.; Adamo, C.; Jaramillo, J.; Gomperts, R.; Stratmann, R. E.; Yazyev, O.; Austin, A. J.; Cammi, R.; Pomelli, C.; Ochterski, J. W.; Martin, R. 
L.; Morokuma, K.; Zakrzewski, V. G.; Voth, G. A.; Salvador, P.; Dannenberg, J. J.; Dapprich, S.; Daniels, A. D.; Farkas, O.; Foresman, J. B.; Ortiz, J. V.; Cioslowski, J. and Fox, D. J. Gaussian 09, Revision B.01, Gaussian, Inc., Wallingford, CT, 2010.

5 Pei, Y., Gao, Y., Zeng, X. C. J. Am. Chem. Soc., 2008, 130, 7830.

6 Pei, Y., Shao, N., Gao, Y., Zeng, X. C. ACS Nano. 2010, 4, 2009.

7 Perdew, J. P.; Ruzsinsky, A.; Tao, J.; Staroverov, V. N.; Scuseria, G. E.; Csonka, G. I. J. Chem. Phys., 2005, 123, 062001.

8 Qian, H., Zhu, Y., Jin, R., ACS Nano. 2009, 3, 3795.

9 Lu, T.; Chen, F. Multiwfn: A Multifunctional Wavefunction Analyzer. J. Comput. Chem., 2012, 33, 580.

10 Liao, L.; Zhou, S.; Dai, Y.; Liu, L.; Yao, C.; Fu, C.; Yang, J.; Wu, Z. J. Am. Chem. Soc., 2015, 137, 9511. 
Table S1. The Kohn-Sham orbitals, energies, and atomic orbital contribution.

\begin{tabular}{|c|c|c|c|c|c|c|c|}
\hline $\begin{array}{c}\text { Orbital } \\
\text { Set }\end{array}$ & Degeneracy & Orbital Numbers & $\begin{array}{l}\text { Ave. Orbital } \\
\text { Energy }(\mathrm{eV})\end{array}$ & $\begin{array}{c}\% \mathrm{Au} \\
(\mathrm{sp})\end{array}$ & $\begin{array}{l}\% \mathrm{Au} \\
(\mathrm{d})\end{array}$ & $\begin{array}{l}\% \mathrm{~S} \\
(\mathrm{sp})\end{array}$ & $\begin{array}{l}\% \mathrm{Cd} \\
(\mathrm{spd})\end{array}$ \\
\hline LUMO+4 & 2 & 466,467 & -2.13 & 0.63 & 0.06 & 0.21 & 0.01 \\
\hline $\mathrm{LUMO}+3$ & 2 & 464,465 & -2.3 & 0.65 & 0.06 & 0.2 & 0.03 \\
\hline LUMO+2 & 1 & 463 & -2.49 & 0.63 & 0.07 & 0.17 & 0.05 \\
\hline LUMO+1 & 1 & 462 & -2.70 & 0.51 & 0.12 & 0.25 & 0.01 \\
\hline LUMO & 2 & 460,461 & -3.34 & 0.52 & 0.15 & 0.21 & 0.05 \\
\hline HOMO & 4 & $456,457,458,459$ & -4.03 & 0.31 & 0.2 & 0.44 & 0.01 \\
\hline HOMO-1 & 1 & 455 & -4.12 & 0.48 & 0.16 & 0.33 & 0.002 \\
\hline HOMO-2 & 2 & 453,454 & -4.28 & 0.43 & 0.003 & 0.47 & 0.002 \\
\hline HOMO-3 & 2 & 451,452 & -4.43 & 0.3 & 0.24 & 0.44 & 0.002 \\
\hline HOMO-4 & 2 & 449,450 & -4.54 & 0.23 & 0.28 & 0.45 & 0.01 \\
\hline HOMO-5 & 2 & 447,448 & -4.66 & 0.5 & 0.20 & 0.24 & 0.02 \\
\hline HOMO-6 & 4 & $443,444,445,446$ & -4.82 & 0.52 & 0.19 & 0.26 & 0.01 \\
\hline HOMO-7 & 4 & $439,440,441,442$ & -4.97 & 0.42 & 0.3 & 0.24 & 0.01 \\
\hline HOMO-8 & 4 & $435,436,437,438$ & -5.14 & 0.48 & 0.24 & 0.22 & 0.02 \\
\hline HOMO-9 & 2 & 433,434 & -5.25 & 0.69 & 0.14 & 0.14 & 0.01 \\
\hline $\begin{array}{c}\text { HOMO- } \\
10\end{array}$ & 3 & $430,431,432$ & -5.4 & 0.79 & 0.1 & 0.08 & 0.01 \\
\hline $\begin{array}{c}\text { HOMO- } \\
11\end{array}$ & 4 & $426,427,428,429$ & -5.59 & 0.71 & 0.17 & 0.08 & 0.02 \\
\hline $\begin{array}{c}\text { HOMO- } \\
12\end{array}$ & 3 & $423,424,425$ & -5.73 & 0.74 & 0.19 & 0.06 & 0.01 \\
\hline $\begin{array}{c}\text { HOMO- } \\
13 \\
\end{array}$ & 3 & $420,421,422$ & -5.92 & 0.12 & 0.84 & 0.03 & 0.01 \\
\hline
\end{tabular}

Orbitals with energy difference less than $\sim 0.1 \mathrm{eV}$ was degenerated. 
Table S2. Excited states, energies, oscillator strengths, and primary orbital-orbital transitions.

\begin{tabular}{|c|c|c|c|c|}
\hline $\begin{array}{l}\text { Excited } \\
\text { states }\end{array}$ & $\begin{array}{c}\text { Average } \\
\text { energy } \\
\text { eV }\end{array}$ & $\begin{array}{c}\text { Average } \\
\text { oscillator } \\
\text { strengths, } \mathrm{f}\end{array}$ & $\begin{array}{l}\text { Primary } \\
\text { transitions }\end{array}$ & Nature of Transitions \\
\hline $71-74$ & 1.86 & 0.005 & $\begin{array}{l}436,437,450,455 \\
459 \rightarrow 460,461 \\
462,464,465,466\end{array}$ & $\begin{array}{l}\mathrm{HOMO} \rightarrow \mathrm{LUMO}+4 \\
\mathrm{HOMO}-1 \rightarrow \text { LUMO+3 } \\
\text { HOMO-4 } \rightarrow \text { LUMO+1 } \\
\text { HOMO-8 } \rightarrow \text { LUMO }\end{array}$ \\
\hline $127-131$ & 2.24 & 0.004 & $\begin{array}{l}428,429,442,451, \\
454 \rightarrow 460,461,462, \\
464,465,466\end{array}$ & $\begin{array}{l}\mathrm{HOMO}-3 \rightarrow \mathrm{LUMO}+3 \\
\mathrm{HOMO}-3 \rightarrow \mathrm{LUMO}+4 \\
\mathrm{HOMO}-7 \rightarrow \mathrm{LUMO}+1 \\
\mathrm{HOMO}-11 \rightarrow \mathrm{LUMO}\end{array}$ \\
\hline 194-198 & 2.62 & 0.018 & $\begin{array}{l}420,423,438,447 \rightarrow \\
460,462,463,467\end{array}$ & $\begin{array}{l}\text { HOMO-5 } \rightarrow \text { LUMO+4 } \\
\text { HOMO-6 } \rightarrow \text { LUMO+3 } \\
\text { HOMO-8 } \rightarrow \text { LUMO+2 } \\
\text { HOMO-9 } \rightarrow \text { LUMO+1 } \\
\text { HOMO-13 } \rightarrow \text { LUMO }\end{array}$ \\
\hline
\end{tabular}


Table S3. Crystal data and structure refinement for $\mathrm{Au}_{24} \mathrm{Cd}(\mathrm{PET})_{18}$.

Empirical formula

Formula weight

Temperature

Wavelength

Crystal system

Space group

Unit cell dimensions

Volume

Z

Density (calculated)

Absorption coefficient

$\mathrm{F}(000)$

Crystal size

Theta range for data collection

Index ranges

Reflections collected

Independent reflections

Completeness to theta $=25.242^{\circ}$

Absorption correction

Max. and min. transmission

Refinement method

Data / restraints / parameters

Goodness-of-fit on $\mathrm{F}^{2}$

Final R indices [I $>2 \operatorname{sigma}(\mathrm{I})]$

$\mathrm{R}$ indices (all data)

Extinction coefficient

Largest diff. peak and hole
C158 H178 Au24 Cd S18 (two $\mathrm{C}_{7} \mathrm{H}_{8}$ enclosed)

7493.67

123(2) K

$0.71073 \AA$

Triclinic

$\mathrm{P}-1$

$\mathrm{a}=15.9937(13) \AA \quad \mathrm{a}=64.9730(10)^{\circ}$.

$\mathrm{b}=17.7756(14) \AA \quad \mathrm{b}=65.3090(10)^{\circ}$.

$\mathrm{c}=18.0174(14) \AA \quad \mathrm{g}=81.1590(10)^{\circ}$.

4215.4(6) $\AA^{3}$

1

$2.952 \mathrm{Mg} / \mathrm{m}^{3}$

$21.177 \mathrm{~mm}^{-1}$

3358

$0.160 \times 0.110 \times 0.015 \mathrm{~mm}^{3}$

1.265 to $25.010^{\circ}$.

$-19<=\mathrm{h}<=19,-21<=\mathrm{k}<=21,-20<=\mathrm{l}<=21$

19912

$14578[\mathrm{R}($ int $)=0.0452]$

$95.5 \%$

Semi-empirical from equivalents

0.226 and 0.080

Full-matrix least-squares on $\mathrm{F}^{2}$

14578 / $481 / 871$

1.076

$\mathrm{R} 1=0.0565, \mathrm{wR} 2=0.1614$

$\mathrm{R} 1=0.0837, \mathrm{wR} 2=0.2309$

$\mathrm{n} / \mathrm{a}$

4.326 and -5.564 e. $\AA^{-3}$ 
Table S4. Atomic coordinates $\left(\mathrm{x} 10^{4}\right)$ and equivalent isotropic displacement parameters $\left(\AA^{2} \times 10^{3}\right)$ for $\mathrm{Au}_{24} \mathrm{Cd}(\mathrm{PET})_{18} . \mathrm{U}(\mathrm{eq})$ is defined as one third of the trace of the orthogonalized $\mathrm{U}^{\mathrm{ij}}$ tensor.

\begin{tabular}{|c|c|c|c|c|}
\hline & $\mathrm{x}$ & $\mathrm{y}$ & z & $\mathrm{U}(\mathrm{eq})$ \\
\hline $\mathrm{Au}(1)$ & 0 & 0 & 10000 & $13(1)$ \\
\hline $\mathrm{Au}(2)$ & $-535(1)$ & $1309(1)$ & $10556(1)$ & $18(1)$ \\
\hline $\mathrm{Au}(3)$ & $1162(1)$ & $438(1)$ & $10562(1)$ & $19(1)$ \\
\hline $\mathrm{Au}(4)$ & $1887(1)$ & $-104(1)$ & $9171(1)$ & $20(1)$ \\
\hline $\mathrm{Au}(5)$ & $705(1)$ & $334(1)$ & $8184(1)$ & $19(1)$ \\
\hline $\mathrm{Au}(6)$ & $-811(1)$ & $1311(1)$ & $9022(1)$ & $21(1)$ \\
\hline $\mathrm{Au}(7)$ & 994(1) & $1489(1)$ & $8830(1)$ & $20(1)$ \\
\hline $\mathrm{Cd}(2)$ & $-535(1)$ & $1309(1)$ & $10556(1)$ & $18(1)$ \\
\hline $\mathrm{Cd}(3)$ & $1162(1)$ & $438(1)$ & $10562(1)$ & $19(1)$ \\
\hline $\mathrm{Cd}(4)$ & $1887(1)$ & $-104(1)$ & $9171(1)$ & $20(1)$ \\
\hline $\mathrm{Cd}(5)$ & $705(1)$ & $334(1)$ & $8184(1)$ & $19(1)$ \\
\hline $\mathrm{Cd}(6)$ & $-811(1)$ & $1311(1)$ & $9022(1)$ & $21(1)$ \\
\hline $\mathrm{Cd}(7)$ & $994(1)$ & $1489(1)$ & $8830(1)$ & $20(1)$ \\
\hline $\mathrm{Au}(8)$ & $-226(1)$ & $3030(1)$ & $8795(1)$ & $29(1)$ \\
\hline $\mathrm{Au}(9)$ & $-151(1)$ & $1010(1)$ & $12216(1)$ & $21(1)$ \\
\hline $\mathrm{Au}(10)$ & $2856(1)$ & $-730(1)$ & $10514(1)$ & $24(1)$ \\
\hline $\mathrm{Au}(11)$ & $2379(1)$ & $-798(1)$ & $7677(1)$ & $24(1)$ \\
\hline $\mathrm{Au}(12)$ & $615(1)$ & $2265(1)$ & $7069(1)$ & $26(1)$ \\
\hline $\mathrm{Au}(13)$ & 2959(1) & $1402(1)$ & $9007(1)$ & $26(1)$ \\
\hline$S(1)$ & $-901(4)$ & $2726(3)$ & $10295(4)$ & $28(1)$ \\
\hline$C(1 \mathrm{~A})$ & $-126(14)$ & $3235(14)$ & $10493(16)$ & $26(5)$ \\
\hline $\mathrm{C}(2 \mathrm{~A})$ & $865(15)$ & $3114(18)$ & $10150(20)$ & $44(7)$ \\
\hline$C(3 \mathrm{~A})$ & $1240(20)$ & $3630(20)$ & $10470(30)$ & $61(9)$ \\
\hline$C(4 A)$ & $1517(19)$ & $3210(20)$ & $11150(20)$ & $56(8)$ \\
\hline$C(5 A)$ & $2070(30)$ & $3770(20)$ & $11180(30)$ & $106(15)$ \\
\hline$C(6 A)$ & 2087(19) & $4640(17)$ & $10730(20)$ & $64(9)$ \\
\hline$C(7 A)$ & $1812(19)$ & $5001(18)$ & $10030(20)$ & $60(8)$ \\
\hline
\end{tabular}




\begin{tabular}{|c|c|c|c|c|}
\hline $\mathrm{C}(8 \mathrm{~A})$ & $1290(20)$ & $4450(20)$ & $9990(30)$ & $78(11)$ \\
\hline $\mathrm{S}(2)$ & $1415(4)$ & $879(4)$ & $11526(4)$ & $28(1)$ \\
\hline $\mathrm{C}(1 \mathrm{~B})$ & $1671(18)$ & $-80(20)$ & $12290(18)$ & $48(6)$ \\
\hline $\mathrm{C}(2 \mathrm{~B})$ & $1943(18)$ & $-80(20)$ & $12994(18)$ & $85(8)$ \\
\hline$C(3 B)$ & $1265(15)$ & $335(15)$ & $13469(13)$ & $94(8)$ \\
\hline$C(4 B)$ & $383(17)$ & $-10(16)$ & $14021(17)$ & $115(12)$ \\
\hline$C(5 B)$ & $-278(15)$ & $410(20)$ & $14487(18)$ & $128(13)$ \\
\hline$C(6 B)$ & $-60(20)$ & $1180(20)$ & 14401(19) & $118(12)$ \\
\hline$C(7 B)$ & $830(20)$ & $1525(15)$ & $13850(20)$ & $119(11)$ \\
\hline $\mathrm{C}(8 \mathrm{~B})$ & $1486(17)$ & $1102(16)$ & $13384(17)$ & $110(10)$ \\
\hline $\mathrm{S}(3)$ & $3242(4)$ & $-621(4)$ & $8350(4)$ & $29(1)$ \\
\hline$C(1 C)$ & $4075(15)$ & $269(16)$ & $7486(18)$ & $35(6)$ \\
\hline$C(2 C)$ & $5016(13)$ & $16(14)$ & $7528(15)$ & $25(5)$ \\
\hline$C(3 C)$ & $5762(15)$ & $652(15)$ & $6839(17)$ & $30(5)$ \\
\hline$C(4 C)$ & $5940(30)$ & $1320(30)$ & $6990(30)$ & $105(15)$ \\
\hline$C(5 C)$ & $6520(20)$ & $1920(20)$ & $6380(30)$ & $71(10)$ \\
\hline$C(6 C)$ & $7000(30)$ & $1880(20)$ & $5660(30)$ & $73(10)$ \\
\hline$C(7 C)$ & $6932(16)$ & $1252(15)$ & $5423(19)$ & $42(7)$ \\
\hline$C(8 C)$ & $6353(19)$ & $589(17)$ & $6024(19)$ & $59(8)$ \\
\hline $\mathrm{S}(4)$ & $838(5)$ & $1138(4)$ & $6690(4)$ & $34(1)$ \\
\hline$C(1 D)$ & $2066(14)$ & $1210(20)$ & $6007(18)$ & $83(8)$ \\
\hline$C(2 D)$ & $2306(18)$ & $1280(30)$ & $5070(20)$ & 103(9) \\
\hline$C(3 D)$ & $3330(16)$ & $1367(16)$ & $4539(18)$ & $156(12)$ \\
\hline$C(4 D)$ & $3603(17)$ & $1929(18)$ & $3660(20)$ & $152(14)$ \\
\hline$C(5 D)$ & 4533(19) & 2061(18) & $3102(15)$ & $121(13)$ \\
\hline$C(6 D)$ & $5190(14)$ & $1632(18)$ & $3430(20)$ & $133(14)$ \\
\hline$C(7 D)$ & $4920(20)$ & 1070(19) & $4320(20)$ & $138(14)$ \\
\hline$C(8 D)$ & $3990(20)$ & $937(18)$ & $4869(15)$ & $155(14)$ \\
\hline$S(5)$ & $2044(4)$ & $-1986(4)$ & $11395(5)$ & $36(2)$ \\
\hline$C(1 E)$ & $2470(20)$ & $-2640(30)$ & $10710(30)$ & $95(13)$ \\
\hline$C(2 E)$ & $3510(20)$ & $-2562(19)$ & $10220(20)$ & $102(14)$ \\
\hline$C(3 E)$ & $3772(16)$ & $-3053(13)$ & $9661(13)$ & $48(7)$ \\
\hline
\end{tabular}




\begin{tabular}{|c|c|c|c|c|}
\hline$C(4 E)$ & $4590(20)$ & $-3432(19)$ & $9670(20)$ & $85(12)$ \\
\hline$C(5 E)$ & $5170(30)$ & $-3950(20)$ & $9290(30)$ & $130(20)$ \\
\hline$C(6 E)$ & $4820(30)$ & $-4120(20)$ & $8780(30)$ & $120(16)$ \\
\hline$C(7 E)$ & $3980(30)$ & $-3770(20)$ & $8720(20)$ & $81(10)$ \\
\hline$C(8 E)$ & $3450(20)$ & $-3236(17)$ & $9149(17)$ & $63(9)$ \\
\hline$S(6)$ & $2202(4)$ & $2452(3)$ & $8260(4)$ & $26(1)$ \\
\hline $\mathrm{C}(1 \mathrm{~F})$ & $2895(16)$ & $2684(15)$ & $7043(17)$ & $32(5)$ \\
\hline $\mathrm{C}(2 \mathrm{~F})$ & $3815(14)$ & 2992(16) & $6748(12)$ & $34(6)$ \\
\hline $\mathrm{C}(3 \mathrm{~F})$ & $4356(12)$ & $3328(11)$ & $5712(9)$ & $42(6)$ \\
\hline $\mathrm{C}(4 \mathrm{~F})$ & $4389(13)$ & $4164(10)$ & $5158(11)$ & $46(7)$ \\
\hline$C(5 F)$ & $4841(15)$ & $4420(9)$ & $4239(10)$ & $78(13)$ \\
\hline$C(6 F)$ & $5259(14)$ & $3840(13)$ & $3876(9)$ & $53(8)$ \\
\hline$C(7 F)$ & $5225(15)$ & $3004(12)$ & $4431(14)$ & $112(16)$ \\
\hline $\mathrm{C}(8 \mathrm{~F})$ & $4774(15)$ & $2748(9)$ & $5349(13)$ & $89(12)$ \\
\hline $\mathrm{S}(7)$ & $440(5)$ & $3479(3)$ & $7272(4)$ & $33(1)$ \\
\hline$C(1 G)$ & $-489(15)$ & $3996(13)$ & $6909(15)$ & $26(5)$ \\
\hline$C(2 G)$ & $-770(20)$ & $4751(18)$ & $7090(20)$ & $52(8)$ \\
\hline$C(3 G)$ & $-1553(15)$ & $5179(15)$ & $6756(17)$ & $33(6)$ \\
\hline$C(4 G)$ & $-2564(19)$ & $5070(18)$ & $7300(20)$ & $56(8)$ \\
\hline$C(5 G)$ & $-3044(18)$ & $5660(20)$ & $6860(20)$ & $70(10)$ \\
\hline$C(6 G)$ & $-2820(20)$ & $6120(20)$ & $5960(20)$ & $94(13)$ \\
\hline$C(7 G)$ & $-1880(20)$ & $6170(20)$ & $5560(30)$ & $68(9)$ \\
\hline $\mathrm{C}(8 \mathrm{G})$ & $-1170(20)$ & $5746(19)$ & $5860(20)$ & $62(9)$ \\
\hline $\mathrm{S}(8)$ & $-1686(4)$ & 1092(3) & $13059(4)$ & $25(1)$ \\
\hline $\mathrm{C}(1 \mathrm{H})$ & $-1917(17)$ & $2203(17)$ & $12800(20)$ & $48(8)$ \\
\hline $\mathrm{C}(2 \mathrm{H})$ & $-1323(15)$ & $2557(13)$ & $13091(15)$ & $24(5)$ \\
\hline $\mathrm{C}(3 \mathrm{H})$ & $-1474(13)$ & $3499(13)$ & $12784(15)$ & $23(5)$ \\
\hline $\mathrm{C}(4 \mathrm{H})$ & $-700(20)$ & $4076(13)$ & $12240(19)$ & $39(7)$ \\
\hline $\mathrm{C}(5 \mathrm{H})$ & $-800(20)$ & $4908(18)$ & $12060(20)$ & $52(8)$ \\
\hline $\mathrm{C}(6 \mathrm{H})$ & $-1670(20)$ & $5200(20)$ & $12360(20)$ & $65(9)$ \\
\hline $\mathrm{C}(7 \mathrm{H})$ & $-2403(19)$ & $4663(15)$ & $12840(20)$ & $48(8)$ \\
\hline $\mathrm{C}(8 \mathrm{H})$ & $-2331(14)$ & $3794(15)$ & $13128(18)$ & $35(6)$ \\
\hline
\end{tabular}




\begin{tabular}{lllcc}
$\mathrm{S}(9)$ & $3841(4)$ & $434(4)$ & $9671(4)$ & $30(1)$ \\
$\mathrm{C}(1 \mathrm{I})$ & $3971(16)$ & $730(16)$ & $10427(18)$ & $33(6)$ \\
$\mathrm{C}(2 \mathrm{I})$ & $4696(14)$ & $1413(14)$ & $10055(18)$ & $31(5)$ \\
$\mathrm{C}(3 \mathrm{I})$ & $4501(19)$ & $2230(20)$ & $9420(20)$ & $49(7)$ \\
$\mathrm{C}(4 \mathrm{I})$ & $3841(17)$ & $2748(15)$ & $9640(20)$ & $47(7)$ \\
$\mathrm{C}(5 \mathrm{I})$ & $3754(15)$ & $3514(15)$ & $9020(17)$ & $45(7)$ \\
$\mathrm{C}(6 \mathrm{I})$ & $4405(17)$ & $3844(17)$ & $8161(19)$ & $44(7)$ \\
$\mathrm{C}(7 \mathrm{I})$ & $5180(20)$ & $3390(20)$ & $7850(30)$ & $70(10)$ \\
$\mathrm{C}(8 \mathrm{I})$ & $5218(17)$ & $2610(19)$ & $8490(20)$ & $53(8)$ \\
$\mathrm{C}(1)$ & $7650(30)$ & $4520(20)$ & $5040(40)$ & $122(18)$ \\
$\mathrm{C}(2)$ & $8105(17)$ & $3729(18)$ & $5360(20)$ & $46(7)$ \\
$\mathrm{C}(3)$ & $8720(20)$ & $3780(20)$ & $4530(20)$ & $64(11)$ \\
$\mathrm{C}(4)$ & $9350(20)$ & $3130(20)$ & $4610(20)$ & $65(10)$ \\
$\mathrm{C}(5)$ & $9465(19)$ & $2580(20)$ & $5320(20)$ & $60(10)$ \\
$\mathrm{C}(6)$ & $8860(20)$ & $2620(20)$ & $6100(30)$ & $76(11)$ \\
$\mathrm{C}(7)$ & $8236(17)$ & $3246(17)$ & $6063(19)$ & $43(7)$ \\
\hline
\end{tabular}


Table S5. Bond lengths $[\AA]$ and angles $\left[^{\circ}\right]$ for $\mathrm{Au}_{24} \mathrm{Cd}(\mathrm{PET})_{18}$.

\begin{tabular}{|c|c|}
\hline $\mathrm{Au}(1)-\mathrm{Au}(4)$ & $2.7687(8)$ \\
\hline $\mathrm{Au}(1)-\mathrm{Cd}(4) \# 1$ & $2.7688(8)$ \\
\hline $\mathrm{Au}(1)-\mathrm{Au}(4) \# 1$ & $2.7688(8)$ \\
\hline $\mathrm{Au}(1)-\mathrm{Cd}(6) \# 1$ & $2.7790(8)$ \\
\hline $\mathrm{Au}(1)-\mathrm{Au}(6) \# 1$ & $2.7790(8)$ \\
\hline $\mathrm{Au}(1)-\mathrm{Au}(6)$ & $2.7790(8)$ \\
\hline $\mathrm{Au}(1)-\mathrm{Au}(7)$ & $2.7834(8)$ \\
\hline $\mathrm{Au}(1)-\mathrm{Cd}(7) \# 1$ & $2.7834(8)$ \\
\hline $\mathrm{Au}(1)-\mathrm{Au}(7) \# 1$ & $2.7834(8)$ \\
\hline $\mathrm{Au}(2)-\mathrm{S}(1)$ & $2.383(5)$ \\
\hline $\mathrm{Au}(2)-\mathrm{Au}(5) \# 1$ & $2.8071(12)$ \\
\hline $\mathrm{Au}(2)-\mathrm{Au}(3)$ & $2.9119(11)$ \\
\hline $\mathrm{Au}(2)-\mathrm{Au}(7)$ & $2.9654(12)$ \\
\hline $\mathrm{Au}(2)-\mathrm{Au}(6)$ & $2.9740(12)$ \\
\hline $\mathrm{Au}(2)-\mathrm{Au}(4) \# 1$ & $3.0248(11)$ \\
\hline $\mathrm{Au}(2)-\mathrm{Au}(9)$ & $3.1136(12)$ \\
\hline $\mathrm{Au}(2)-\mathrm{Au}(11) \# 1$ & $3.1933(12)$ \\
\hline$A u(2)-A u(8)$ & $3.2573(12)$ \\
\hline $\mathrm{Au}(3)-\mathrm{S}(2)$ & $2.374(5)$ \\
\hline $\mathrm{Au}(3)-\mathrm{Au}(4)$ & $2.7970(12)$ \\
\hline $\mathrm{Au}(3)-\mathrm{Au}(6) \# 1$ & $2.9581(12)$ \\
\hline $\mathrm{Au}(3)-\mathrm{Au}(5) \# 1$ & $2.9829(12)$ \\
\hline $\mathrm{Au}(3)-\mathrm{Au}(7)$ & $2.9858(13)$ \\
\hline $\mathrm{Au}(3)-\mathrm{Au}(13)$ & $3.1270(12)$ \\
\hline $\mathrm{Au}(3)-\mathrm{Au}(10)$ & $3.1439(11)$ \\
\hline $\mathrm{Au}(3)-\mathrm{Au}(9)$ & $3.3163(12)$ \\
\hline $\mathrm{Au}(4)-\mathrm{S}(3)$ & $2.365(5)$ \\
\hline $\mathrm{Au}(4)-\mathrm{Au}(7)$ & $2.9070(12)$ \\
\hline $\mathrm{Au}(4)-\mathrm{Au}(5)$ & $2.9205(12)$ \\
\hline $\mathrm{Au}(4)-\mathrm{Au}(6) \# 1$ & $2.9509(13)$ \\
\hline
\end{tabular}




\begin{tabular}{|c|c|}
\hline $\mathrm{Au}(4)-\mathrm{Au}(2) \# 1$ & $3.0248(11)$ \\
\hline $\mathrm{Au}(4)-\mathrm{Au}(10)$ & $3.1338(12)$ \\
\hline $\mathrm{Au}(4)-\mathrm{Au}(11)$ & $3.1826(12)$ \\
\hline $\mathrm{Au}(4)-\mathrm{Au}(13)$ & $3.2417(11)$ \\
\hline $\mathrm{Au}(5)-\mathrm{S}(4)$ & $2.385(6)$ \\
\hline $\mathrm{Au}(5)-\mathrm{Au}(2) \# 1$ & $2.8071(12)$ \\
\hline $\mathrm{Au}(5)-\mathrm{Au}(7)$ & $2.9232(11)$ \\
\hline $\mathrm{Au}(5)-\mathrm{Au}(3) \# 1$ & $2.9829(12)$ \\
\hline $\mathrm{Au}(5)-\mathrm{Au}(6)$ & $3.0055(12)$ \\
\hline $\mathrm{Au}(5)-\mathrm{Au}(9) \# 1$ & $3.1060(11)$ \\
\hline $\mathrm{Au}(5)-\mathrm{Au}(11)$ & $3.1484(12)$ \\
\hline $\mathrm{Au}(5)-\mathrm{Au}(12)$ & $3.1762(12)$ \\
\hline $\mathrm{Au}(6)-\mathrm{S}(5) \# 1$ & $2.375(6)$ \\
\hline $\mathrm{Au}(6)-\mathrm{Au}(7)$ & $2.8115(11)$ \\
\hline $\mathrm{Au}(6)-\mathrm{Au}(4) \# 1$ & $2.9509(13)$ \\
\hline $\mathrm{Au}(6)-\mathrm{Au}(3) \# 1$ & $2.9581(12)$ \\
\hline $\mathrm{Au}(6)-\mathrm{Au}(8)$ & $3.1430(12)$ \\
\hline $\mathrm{Au}(6)-\mathrm{Au}(12)$ & $3.1451(13)$ \\
\hline $\mathrm{Au}(6)-\mathrm{Au}(10) \# 1$ & $3.2377(11)$ \\
\hline $\mathrm{Au}(7)-\mathrm{S}(6)$ & $2.365(5)$ \\
\hline $\operatorname{Au}(7)-A u(8)$ & $3.1019(12)$ \\
\hline $\mathrm{Au}(7)-\mathrm{Au}(12)$ & $3.1597(13)$ \\
\hline $\mathrm{Au}(7)-\mathrm{Au}(13)$ & $3.2640(12)$ \\
\hline $\mathrm{Au}(8)-\mathrm{S}(7)$ & $2.294(7)$ \\
\hline $\mathrm{Au}(8)-\mathrm{S}(1)$ & $2.296(7)$ \\
\hline $\mathrm{Au}(9)-\mathrm{S}(8)$ & $2.306(6)$ \\
\hline $\mathrm{Au}(9)-\mathrm{S}(2)$ & $2.311(5)$ \\
\hline $\mathrm{Au}(9)-\mathrm{Cd}(5) \# 1$ & $3.1060(11)$ \\
\hline $\mathrm{Au}(9)-\mathrm{Au}(5) \# 1$ & $3.1060(11)$ \\
\hline $\mathrm{Au}(10)-\mathrm{S}(5)$ & $2.316(7)$ \\
\hline $\mathrm{Au}(10)-\mathrm{S}(9)$ & $2.327(6)$ \\
\hline $\mathrm{Au}(10)-\mathrm{Cd}(6) \# 1$ & $3.2378(11)$ \\
\hline
\end{tabular}




\begin{tabular}{|c|c|}
\hline $\mathrm{Au}(10)-\mathrm{Au}(6) \# 1$ & $3.2378(11)$ \\
\hline $\operatorname{Au}(11)-S(8) \# 1$ & $2.292(6)$ \\
\hline $\mathrm{Au}(11)-\mathrm{S}(3)$ & $2.308(6)$ \\
\hline $\mathrm{Au}(11)-\mathrm{Au}(2) \# 1$ & $3.1933(12)$ \\
\hline $\mathrm{Au}(11)-\mathrm{Cd}(2) \# 1$ & $3.1933(12)$ \\
\hline $\mathrm{Au}(12)-\mathrm{S}(7)$ & $2.295(6)$ \\
\hline $\mathrm{Au}(12)-\mathrm{S}(4)$ & $2.311(6)$ \\
\hline $\mathrm{Au}(13)-\mathrm{S}(9)$ & $2.302(6)$ \\
\hline $\mathrm{Au}(13)-\mathrm{S}(6)$ & $2.304(6)$ \\
\hline$S(1)-C(1 A)$ & $1.86(2)$ \\
\hline$C(1 \mathrm{~A})-\mathrm{C}(2 \mathrm{~A})$ & $1.46(3)$ \\
\hline$C(2 A)-C(3 A)$ & $1.56(3)$ \\
\hline$C(3 A)-C(8 A)$ & $1.34(5)$ \\
\hline$C(3 A)-C(4 A)$ & $1.37(5)$ \\
\hline$C(4 A)-C(5 A)$ & $1.45(5)$ \\
\hline$C(5 A)-C(6 A)$ & $1.411(19)$ \\
\hline$C(6 A)-C(7 A)$ & $1.384(19)$ \\
\hline$C(7 A)-C(8 A)$ & $1.413(18)$ \\
\hline $\mathrm{S}(2)-\mathrm{C}(1 \mathrm{~B})$ & $1.80(3)$ \\
\hline$C(1 B)-C(2 B)$ & $1.510(18)$ \\
\hline $\mathrm{C}(2 \mathrm{~B})-\mathrm{C}(3 \mathrm{~B})$ & $1.398(9)$ \\
\hline$C(3 B)-C(4 B)$ & 1.3900 \\
\hline $\mathrm{C}(3 \mathrm{~B})-\mathrm{C}(8 \mathrm{~B})$ & 1.3900 \\
\hline$C(4 B)-C(5 B)$ & 1.3900 \\
\hline $\mathrm{C}(5 \mathrm{~B})-\mathrm{C}(6 \mathrm{~B})$ & 1.3900 \\
\hline$C(6 B)-C(7 B)$ & 1.3900 \\
\hline$C(7 B)-C(8 B)$ & 1.3900 \\
\hline $\mathrm{S}(3)-\mathrm{C}(1 \mathrm{C})$ & $1.88(2)$ \\
\hline $\mathrm{C}(1 \mathrm{C})-\mathrm{C}(2 \mathrm{C})$ & $1.53(3)$ \\
\hline $\mathrm{C}(2 \mathrm{C})-\mathrm{C}(3 \mathrm{C})$ & $1.49(3)$ \\
\hline$C(3 C)-C(8 C)$ & $1.42(4)$ \\
\hline$C(3 C)-C(4 C)$ & $1.42(5)$ \\
\hline
\end{tabular}




\begin{tabular}{|c|c|}
\hline$C(4 C)-C(5 C)$ & $1.28(5)$ \\
\hline$C(5 C)-C(6 C)$ & $1.23(5)$ \\
\hline$C(6 C)-C(7 C)$ & $1.39(4)$ \\
\hline$C(7 C)-C(8 C)$ & $1.354(18)$ \\
\hline $\mathrm{S}(4)-\mathrm{C}(1 \mathrm{D})$ & $1.820(17)$ \\
\hline$C(1 D)-C(2 D)$ & $1.519(19)$ \\
\hline $\mathrm{C}(2 \mathrm{D})-\mathrm{C}(3 \mathrm{D})$ & $1.505(19)$ \\
\hline$C(3 D)-C(4 D)$ & 1.3900 \\
\hline$C(3 D)-C(8 D)$ & 1.3900 \\
\hline$C(4 D)-C(5 D)$ & 1.3900 \\
\hline$C(5 D)-C(6 D)$ & 1.3900 \\
\hline$C(6 D)-C(7 D)$ & 1.3900 \\
\hline$C(7 D)-C(8 D)$ & 1.3900 \\
\hline$S(5)-C(1 E)$ & $1.90(4)$ \\
\hline $\mathrm{S}(5)-\mathrm{Cd}(6) \# 1$ & $2.375(6)$ \\
\hline $\mathrm{S}(5)-\mathrm{Au}(6) \# 1$ & $2.375(6)$ \\
\hline$C(1 E)-C(2 E)$ & $1.514(19)$ \\
\hline$C(2 E)-C(3 E)$ & $1.498(19)$ \\
\hline$C(3 E)-C(4 E)$ & $1.38(4)$ \\
\hline$C(3 E)-C(8 E)$ & $1.388(19)$ \\
\hline$C(4 E)-C(5 E)$ & $1.39(2)$ \\
\hline$C(5 E)-C(6 E)$ & $1.40(2)$ \\
\hline$C(6 E)-C(7 E)$ & $1.41(5)$ \\
\hline$C(7 E)-C(8 E)$ & $1.43(5)$ \\
\hline$S(6)-C(1 F)$ & $1.88(3)$ \\
\hline$C(1 F)-C(2 F)$ & $1.45(3)$ \\
\hline $\mathrm{C}(2 \mathrm{~F})-\mathrm{C}(3 \mathrm{~F})$ & $1.566(17)$ \\
\hline$C(3 F)-C(4 F)$ & 1.3900 \\
\hline $\mathrm{C}(3 \mathrm{~F})-\mathrm{C}(8 \mathrm{~F})$ & 1.3900 \\
\hline$C(4 F)-C(5 F)$ & 1.3900 \\
\hline $\mathrm{C}(5 \mathrm{~F})-\mathrm{C}(6 \mathrm{~F})$ & 1.3900 \\
\hline$C(6 F)-C(7 F)$ & 1.3900 \\
\hline
\end{tabular}




\begin{tabular}{|c|c|}
\hline $\mathrm{C}(7 \mathrm{~F})-\mathrm{C}(8 \mathrm{~F})$ & 1.3900 \\
\hline$S(7)-C(1 G)$ & $1.83(2)$ \\
\hline$C(1 G)-C(2 G)$ & $1.47(3)$ \\
\hline$C(2 G)-C(3 G)$ & $1.57(4)$ \\
\hline$C(3 G)-C(8 G)$ & $1.41(4)$ \\
\hline$C(3 G)-C(4 G)$ & $1.49(4)$ \\
\hline$C(4 G)-C(5 G)$ & $1.378(19)$ \\
\hline$C(5 G)-C(6 G)$ & $1.383(19)$ \\
\hline$C(6 G)-C(7 G)$ & $1.370(19)$ \\
\hline$C(7 G)-C(8 G)$ & $1.43(4)$ \\
\hline $\mathrm{S}(8)-\mathrm{C}(1 \mathrm{H})$ & $1.84(3)$ \\
\hline $\mathrm{S}(8)-\mathrm{Au}(11) \# 1$ & $2.292(6)$ \\
\hline $\mathrm{C}(1 \mathrm{H})-\mathrm{C}(2 \mathrm{H})$ & $1.57(3)$ \\
\hline $\mathrm{C}(2 \mathrm{H})-\mathrm{C}(3 \mathrm{H})$ & $1.54(3)$ \\
\hline $\mathrm{C}(3 \mathrm{H})-\mathrm{C}(8 \mathrm{H})$ & $1.371(17)$ \\
\hline $\mathrm{C}(3 \mathrm{H})-\mathrm{C}(4 \mathrm{H})$ & $1.43(3)$ \\
\hline $\mathrm{C}(4 \mathrm{H})-\mathrm{C}(5 \mathrm{H})$ & $1.37(4)$ \\
\hline $\mathrm{C}(5 \mathrm{H})-\mathrm{C}(6 \mathrm{H})$ & $1.38(4)$ \\
\hline $\mathrm{C}(6 \mathrm{H})-\mathrm{C}(7 \mathrm{H})$ & $1.35(4)$ \\
\hline $\mathrm{C}(7 \mathrm{H})-\mathrm{C}(8 \mathrm{H})$ & $1.41(3)$ \\
\hline $\mathrm{S}(9)-\mathrm{C}(1 \mathrm{I})$ & $1.75(2)$ \\
\hline $\mathrm{C}(1 \mathrm{I})-\mathrm{C}(2 \mathrm{I})$ & $1.54(3)$ \\
\hline $\mathrm{C}(2 \mathrm{I})-\mathrm{C}(3 \mathrm{I})$ & $1.50(4)$ \\
\hline$C(3 \mathrm{I})-\mathrm{C}(4 \mathrm{I})$ & $1.34(4)$ \\
\hline $\mathrm{C}(3 \mathrm{I})-\mathrm{C}(8 \mathrm{I})$ & $1.50(5)$ \\
\hline$C(4 \mathrm{I})-\mathrm{C}(5 \mathrm{I})$ & $1.381(19)$ \\
\hline $\mathrm{C}(5 \mathrm{I})-\mathrm{C}(6 \mathrm{I})$ & $1.38(4)$ \\
\hline $\mathrm{C}(6 \mathrm{I})-\mathrm{C}(7 \mathrm{I})$ & $1.411(19)$ \\
\hline $\mathrm{C}(7 \mathrm{I})-\mathrm{C}(8 \mathrm{I})$ & $1.40(5)$ \\
\hline$C(1)-C(2)$ & $1.475(19)$ \\
\hline$C(2)-C(7)$ & $1.27(4)$ \\
\hline$C(2)-C(3)$ & $1.38(4)$ \\
\hline
\end{tabular}




\begin{tabular}{|c|c|}
\hline$C(3)-C(4)$ & $1.41(5)$ \\
\hline$C(4)-C(5)$ & $1.31(4)$ \\
\hline$C(5)-C(6)$ & $1.36(5)$ \\
\hline$C(6)-C(7)$ & $1.372(19)$ \\
\hline $\mathrm{Au}(4)-\mathrm{Au}(1)-\mathrm{Au}(4) \# 1$ & 180.0 \\
\hline $\mathrm{Cd}(4) \# 1-\mathrm{Au}(1)-\mathrm{Cd}(6) \# 1$ & $115.73(3)$ \\
\hline $\mathrm{Au}(4)-\mathrm{Au}(1)-\mathrm{Au}(6) \# 1$ & $64.27(3)$ \\
\hline $\mathrm{Au}(4) \# 1-\mathrm{Au}(1)-\mathrm{Au}(6) \# 1$ & $115.73(3)$ \\
\hline$A u(4)-A u(1)-A u(6)$ & $115.73(3)$ \\
\hline $\mathrm{Au}(4) \# 1-\mathrm{Au}(1)-\mathrm{Au}(6)$ & $64.27(3)$ \\
\hline $\mathrm{Au}(6) \# 1-\mathrm{Au}(1)-\mathrm{Au}(6)$ & $180.00(3)$ \\
\hline $\mathrm{Au}(4)-\mathrm{Au}(1)-\mathrm{Au}(7)$ & $63.15(3)$ \\
\hline $\mathrm{Au}(4) \# 1-\mathrm{Au}(1)-\mathrm{Au}(7)$ & $116.85(2)$ \\
\hline $\mathrm{Au}(6) \# 1-\mathrm{Au}(1)-\mathrm{Au}(7)$ & $119.28(2)$ \\
\hline $\mathrm{Au}(6)-\mathrm{Au}(1)-\mathrm{Au}(7)$ & $60.72(2)$ \\
\hline $\mathrm{Cd}(4) \# 1-\mathrm{Au}(1)-\mathrm{Cd}(7) \# 1$ & $63.15(2)$ \\
\hline $\mathrm{Cd}(6) \# 1-\mathrm{Au}(1)-\mathrm{Cd}(7) \# 1$ & $60.72(2)$ \\
\hline $\mathrm{Au}(4)-\mathrm{Au}(1)-\mathrm{Au}(7) \# 1$ & $116.85(2)$ \\
\hline $\mathrm{Au}(4) \# 1-\mathrm{Au}(1)-\mathrm{Au}(7) \# 1$ & $63.15(2)$ \\
\hline $\mathrm{Au}(6) \# 1-\mathrm{Au}(1)-\mathrm{Au}(7) \# 1$ & $60.72(2)$ \\
\hline $\mathrm{Au}(6)-\mathrm{Au}(1)-\mathrm{Au}(7) \# 1$ & $119.28(2)$ \\
\hline $\mathrm{Au}(7)-\mathrm{Au}(1)-\mathrm{Au}(7) \# 1$ & 180.0 \\
\hline $\mathrm{S}(1)-\mathrm{Au}(2)-\mathrm{Au}(1)$ & $152.04(16)$ \\
\hline $\mathrm{S}(1)-\mathrm{Au}(2)-\mathrm{Au}(5) \# 1$ & 146.97(16) \\
\hline $\mathrm{Au}(1)-\mathrm{Au}(2)-\mathrm{Au}(5) \# 1$ & $59.89(2)$ \\
\hline $\mathrm{S}(1)-\mathrm{Au}(2)-\mathrm{Au}(3)$ & $130.63(14)$ \\
\hline$A u(1)-A u(2)-A u(3)$ & $58.45(2)$ \\
\hline $\mathrm{Au}(5) \# 1-\mathrm{Au}(2)-\mathrm{Au}(3)$ & $62.84(3)$ \\
\hline $\mathrm{S}(1)-\mathrm{Au}(2)-\mathrm{Au}(7)$ & $100.95(16)$ \\
\hline $\mathrm{Au}(1)-\mathrm{Au}(2)-\mathrm{Au}(7)$ & $57.73(2)$ \\
\hline $\mathrm{Au}(5) \# 1-\mathrm{Au}(2)-\mathrm{Au}(7)$ & $110.74(3)$ \\
\hline
\end{tabular}




\begin{tabular}{|c|c|}
\hline $\operatorname{Au}(3)-A u(2)-A u(7)$ & $61.06(3)$ \\
\hline $\mathrm{S}(1)-\mathrm{Au}(2)-\mathrm{Au}(6)$ & $96.39(15)$ \\
\hline$A u(1)-A u(2)-A u(6)$ & $57.53(2)$ \\
\hline $\mathrm{Au}(5) \# 1-\mathrm{Au}(2)-\mathrm{Au}(6)$ & $108.54(3)$ \\
\hline$A u(3)-A u(2)-A u(6)$ & $106.45(3)$ \\
\hline$A u(7)-A u(2)-A u(6)$ & $56.51(3)$ \\
\hline $\mathrm{S}(1)-\mathrm{Au}(2)-\mathrm{Au}(4) \# 1$ & $121.35(14)$ \\
\hline $\mathrm{Au}(1)-\mathrm{Au}(2)-\mathrm{Au}(4) \# 1$ & $56.68(2)$ \\
\hline $\mathrm{Au}(5) \# 1-\mathrm{Au}(2)-\mathrm{Au}(4) \# 1$ & $59.97(3)$ \\
\hline$A u(3)-A u(2)-A u(4) \# 1$ & $107.90(3)$ \\
\hline$A u(7)-A u(2)-A u(4) \# 1$ & $104.31(3)$ \\
\hline$A u(6)-A u(2)-A u(4) \# 1$ & $58.93(3)$ \\
\hline $\mathrm{S}(1)-\mathrm{Au}(2)-\mathrm{Au}(9)$ & $92.94(15)$ \\
\hline $\mathrm{Au}(1)-\mathrm{Au}(2)-\mathrm{Au}(9)$ & $113.22(3)$ \\
\hline $\mathrm{Au}(5) \# 1-\mathrm{Au}(2)-\mathrm{Au}(9)$ & $63.03(3)$ \\
\hline$A u(3)-A u(2)-A u(9)$ & $66.69(3)$ \\
\hline$A u(7)-A u(2)-A u(9)$ & $121.16(3)$ \\
\hline $\mathrm{Au}(6)-\mathrm{Au}(2)-\mathrm{Au}(9)$ & $170.67(4)$ \\
\hline $\mathrm{Au}(4) \# 1-\mathrm{Au}(2)-\mathrm{Au}(9)$ & $116.06(4)$ \\
\hline $\mathrm{S}(1)-\mathrm{Au}(2)-\mathrm{Au}(11) \# 1$ & $88.08(16)$ \\
\hline $\mathrm{Au}(1)-\mathrm{Au}(2)-\mathrm{Au}(11) \# 1$ & 109.93(3) \\
\hline $\mathrm{Au}(5) \# 1-\mathrm{Au}(2)-\mathrm{Au}(11) \# 1$ & $62.91(3)$ \\
\hline $\mathrm{Au}(3)-\mathrm{Au}(2)-\mathrm{Au}(11) \# 1$ & $120.59(4)$ \\
\hline $\operatorname{Au}(7)-A u(2)-A u(11) \# 1$ & $165.80(3)$ \\
\hline $\mathrm{Au}(6)-\mathrm{Au}(2)-\mathrm{Au}(11) \# 1$ & $111.99(3)$ \\
\hline $\mathrm{Au}(4) \# 1-\mathrm{Au}(2)-\mathrm{Au}(11) \# 1$ & $61.50(3)$ \\
\hline $\mathrm{Au}(9)-\mathrm{Au}(2)-\mathrm{Au}(11) \# 1$ & $68.67(3)$ \\
\hline $\mathrm{S}(1)-\mathrm{Au}(2)-\mathrm{Au}(8)$ & $44.78(16)$ \\
\hline $\mathrm{Au}(1)-\mathrm{Au}(2)-\mathrm{Au}(8)$ & $107.96(3)$ \\
\hline $\mathrm{Au}(5) \# 1-\mathrm{Au}(2)-\mathrm{Au}(8)$ & $167.75(4)$ \\
\hline $\operatorname{Au}(3)-A u(2)-A u(8)$ & $113.45(4)$ \\
\hline$A u(7)-A u(2)-A u(8)$ & $59.58(3)$ \\
\hline
\end{tabular}




\begin{tabular}{|c|c|}
\hline$A u(6)-A u(2)-A u(8)$ & $60.38(3)$ \\
\hline $\mathrm{Au}(4) \# 1-\mathrm{Au}(2)-\mathrm{Au}(8)$ & $113.20(3)$ \\
\hline $\mathrm{Au}(9)-\mathrm{Au}(2)-\mathrm{Au}(8)$ & $127.54(3)$ \\
\hline $\mathrm{Au}(11) \# 1-\mathrm{Au}(2)-\mathrm{Au}(8)$ & $124.50(3)$ \\
\hline$S(2)-A u(3)-A u(1)$ & $151.68(14)$ \\
\hline $\mathrm{S}(2)-\mathrm{Au}(3)-\mathrm{Au}(4)$ & $148.81(14)$ \\
\hline $\mathrm{Au}(1)-\mathrm{Au}(3)-\mathrm{Au}(4)$ & $59.45(2)$ \\
\hline$S(2)-A u(3)-A u(2)$ & $98.09(14)$ \\
\hline$A u(1)-A u(3)-A u(2)$ & $58.61(2)$ \\
\hline$A u(4)-A u(3)-A u(2)$ & $109.60(3)$ \\
\hline $\mathrm{S}(2)-\mathrm{Au}(3)-\mathrm{Au}(6) \# 1$ & $124.14(16)$ \\
\hline $\mathrm{Au}(1)-\mathrm{Au}(3)-\mathrm{Au}(6) \# 1$ & $57.77(2)$ \\
\hline$A u(4)-A u(3)-A u(6) \# 1$ & $61.62(3)$ \\
\hline$A u(2)-A u(3)-A u(6) \# 1$ & $106.72(3)$ \\
\hline $\mathrm{S}(2)-\mathrm{Au}(3)-\mathrm{Au}(5) \# 1$ & $96.91(14)$ \\
\hline$A u(1)-A u(3)-A u(5) \# 1$ & $57.82(2)$ \\
\hline $\mathrm{Au}(4)-\mathrm{Au}(3)-\mathrm{Au}(5) \# 1$ & $109.98(3)$ \\
\hline$A u(2)-A u(3)-A u(5) \# 1$ & $56.86(3)$ \\
\hline $\mathrm{Au}(6) \# 1-\mathrm{Au}(3)-\mathrm{Au}(5) \# 1$ & $60.78(3)$ \\
\hline $\mathrm{S}(2)-\mathrm{Au}(3)-\mathrm{Au}(7)$ & $128.01(16)$ \\
\hline $\operatorname{Au}(1)-A u(3)-A u(7)$ & $57.53(2)$ \\
\hline$A u(4)-A u(3)-A u(7)$ & $60.25(3)$ \\
\hline $\operatorname{Au}(2)-A u(3)-A u(7)$ & $60.36(3)$ \\
\hline $\mathrm{Au}(6) \# 1-\mathrm{Au}(3)-\mathrm{Au}(7)$ & $107.70(3)$ \\
\hline $\mathrm{Au}(5) \# 1-\mathrm{Au}(3)-\mathrm{Au}(7)$ & $105.49(3)$ \\
\hline$S(2)-A u(3)-A u(13)$ & $89.71(14)$ \\
\hline $\mathrm{Au}(1)-\mathrm{Au}(3)-\mathrm{Au}(13)$ & $113.84(3)$ \\
\hline$A u(4)-A u(3)-A u(13)$ & $66.08(3)$ \\
\hline$A u(2)-A u(3)-A u(13)$ & $114.86(4)$ \\
\hline $\mathrm{Au}(6) \# 1-\mathrm{Au}(3)-\mathrm{Au}(13)$ & $121.08(4)$ \\
\hline $\mathrm{Au}(5) \# 1-\mathrm{Au}(3)-\mathrm{Au}(13)$ & $169.98(4)$ \\
\hline $\mathrm{Au}(7)-\mathrm{Au}(3)-\mathrm{Au}(13)$ & $64.50(3)$ \\
\hline
\end{tabular}




\begin{tabular}{|c|c|}
\hline $\mathrm{S}(2)-\mathrm{Au}(3)-\mathrm{Au}(10)$ & $90.65(14)$ \\
\hline$A u(1)-A u(3)-A u(10)$ & $111.59(3)$ \\
\hline$A u(4)-A u(3)-A u(10)$ & $63.36(3)$ \\
\hline$A u(2)-A u(3)-A u(10)$ & $170.00(4)$ \\
\hline $\mathrm{Au}(6) \# 1-\mathrm{Au}(3)-\mathrm{Au}(10)$ & $64.01(3)$ \\
\hline $\mathrm{Au}(5) \# 1-\mathrm{Au}(3)-\mathrm{Au}(10)$ & $117.44(4)$ \\
\hline $\mathrm{Au}(7)-\mathrm{Au}(3)-\mathrm{Au}(10)$ & $117.42(4)$ \\
\hline $\mathrm{Au}(13)-\mathrm{Au}(3)-\mathrm{Au}(10)$ & $69.80(3)$ \\
\hline $\mathrm{S}(2)-\mathrm{Au}(3)-\mathrm{Au}(9)$ & $44.16(13)$ \\
\hline $\mathrm{Au}(1)-\mathrm{Au}(3)-\mathrm{Au}(9)$ & $107.56(3)$ \\
\hline $\mathrm{Au}(4)-\mathrm{Au}(3)-\mathrm{Au}(9)$ & $167.01(3)$ \\
\hline $\mathrm{Au}(2)-\mathrm{Au}(3)-\mathrm{Au}(9)$ & $59.57(3)$ \\
\hline $\mathrm{Au}(6) \# 1-\mathrm{Au}(3)-\mathrm{Au}(9)$ & $112.69(3)$ \\
\hline $\mathrm{Au}(5) \# 1-\mathrm{Au}(3)-\mathrm{Au}(9)$ & $58.80(3)$ \\
\hline $\mathrm{Au}(7)-\mathrm{Au}(3)-\mathrm{Au}(9)$ & $114.24(3)$ \\
\hline $\mathrm{Au}(13)-\mathrm{Au}(3)-\mathrm{Au}(9)$ & $123.73(3)$ \\
\hline $\mathrm{Au}(10)-\mathrm{Au}(3)-\mathrm{Au}(9)$ & $126.27(3)$ \\
\hline $\mathrm{S}(3)-\mathrm{Au}(4)-\mathrm{Au}(1)$ & $154.54(14)$ \\
\hline $\mathrm{S}(3)-\mathrm{Au}(4)-\mathrm{Au}(3)$ & $142.00(14)$ \\
\hline $\mathrm{Au}(1)-\mathrm{Au}(4)-\mathrm{Au}(3)$ & $60.09(2)$ \\
\hline $\mathrm{S}(3)-\mathrm{Au}(4)-\mathrm{Au}(7)$ & $134.90(16)$ \\
\hline $\mathrm{Au}(1)-\mathrm{Au}(4)-\mathrm{Au}(7)$ & $58.67(2)$ \\
\hline $\mathrm{Au}(3)-\mathrm{Au}(4)-\mathrm{Au}(7)$ & $63.09(3)$ \\
\hline$S(3)-A u(4)-A u(5)$ & $105.93(15)$ \\
\hline $\mathrm{Au}(1)-\mathrm{Au}(4)-\mathrm{Au}(5)$ & $58.77(2)$ \\
\hline $\mathrm{Au}(3)-\mathrm{Au}(4)-\mathrm{Au}(5)$ & $111.18(3)$ \\
\hline$A u(7)-A u(4)-A u(5)$ & $60.21(3)$ \\
\hline $\mathrm{S}(3)-\mathrm{Au}(4)-\mathrm{Au}(6) \# 1$ & $115.06(16)$ \\
\hline $\mathrm{Au}(1)-\mathrm{Au}(4)-\mathrm{Au}(6) \# 1$ & $58.03(2)$ \\
\hline $\mathrm{Au}(3)-\mathrm{Au}(4)-\mathrm{Au}(6) \# 1$ & $61.88(3)$ \\
\hline $\mathrm{Au}(7)-\mathrm{Au}(4)-\mathrm{Au}(6) \# 1$ & $110.03(4)$ \\
\hline$A u(5)-A u(4)-A u(6) \# 1$ & $106.15(3)$ \\
\hline
\end{tabular}




\begin{tabular}{|c|c|}
\hline $\mathrm{S}(3)-\mathrm{Au}(4)-\mathrm{Au}(2) \# 1$ & $97.41(14)$ \\
\hline $\mathrm{Au}(1)-\mathrm{Au}(4)-\mathrm{Au}(2) \# 1$ & $57.41(2)$ \\
\hline $\mathrm{Au}(3)-\mathrm{Au}(4)-\mathrm{Au}(2) \# 1$ & $109.57(4)$ \\
\hline $\mathrm{Au}(7)-\mathrm{Au}(4)-\mathrm{Au}(2) \# 1$ & $105.45(3)$ \\
\hline $\mathrm{Au}(5)-\mathrm{Au}(4)-\mathrm{Au}(2) \# 1$ & $56.32(3)$ \\
\hline $\mathrm{Au}(6) \# 1-\mathrm{Au}(4)-\mathrm{Au}(2) \# 1$ & $59.68(3)$ \\
\hline$S(3)-A u(4)-A u(10)$ & $80.65(15)$ \\
\hline $\mathrm{Au}(1)-\mathrm{Au}(4)-\mathrm{Au}(10)$ & $112.39(3)$ \\
\hline $\mathrm{Au}(3)-\mathrm{Au}(4)-\mathrm{Au}(10)$ & $63.73(3)$ \\
\hline $\mathrm{Au}(7)-\mathrm{Au}(4)-\mathrm{Au}(10)$ & $120.23(4)$ \\
\hline $\mathrm{Au}(5)-\mathrm{Au}(4)-\mathrm{Au}(10)$ & $170.25(4)$ \\
\hline $\mathrm{Au}(6) \# 1-\mathrm{Au}(4)-\mathrm{Au}(10)$ & $64.22(3)$ \\
\hline $\mathrm{Au}(2) \# 1-\mathrm{Au}(4)-\mathrm{Au}(10)$ & $116.35(4)$ \\
\hline$S(3)-A u(4)-A u(11)$ & $46.33(14)$ \\
\hline $\mathrm{Au}(1)-\mathrm{Au}(4)-\mathrm{Au}(11)$ & $110.85(3)$ \\
\hline $\mathrm{Au}(3)-\mathrm{Au}(4)-\mathrm{Au}(11)$ & $170.82(4)$ \\
\hline $\mathrm{Au}(7)-\mathrm{Au}(4)-\mathrm{Au}(11)$ & $114.59(4)$ \\
\hline $\mathrm{Au}(5)-\mathrm{Au}(4)-\mathrm{Au}(11)$ & $61.94(3)$ \\
\hline $\mathrm{Au}(6) \# 1-\mathrm{Au}(4)-\mathrm{Au}(11)$ & $112.92(3)$ \\
\hline $\mathrm{Au}(2) \# 1-\mathrm{Au}(4)-\mathrm{Au}(11)$ & $61.86(3)$ \\
\hline $\mathrm{Au}(10)-\mathrm{Au}(4)-\mathrm{Au}(11)$ & $122.04(3)$ \\
\hline $\mathrm{S}(3)-\mathrm{Au}(4)-\mathrm{Au}(13)$ & $94.05(14)$ \\
\hline $\mathrm{Au}(1)-\mathrm{Au}(4)-\mathrm{Au}(13)$ & $110.94(3)$ \\
\hline $\mathrm{Au}(3)-\mathrm{Au}(4)-\mathrm{Au}(13)$ & $61.86(3)$ \\
\hline $\mathrm{Au}(7)-\mathrm{Au}(4)-\mathrm{Au}(13)$ & $63.85(3)$ \\
\hline $\mathrm{Au}(5)-\mathrm{Au}(4)-\mathrm{Au}(13)$ & $117.40(4)$ \\
\hline $\mathrm{Au}(6) \# 1-\mathrm{Au}(4)-\mathrm{Au}(13)$ & $117.59(4)$ \\
\hline $\mathrm{Au}(2) \# 1-\mathrm{Au}(4)-\mathrm{Au}(13)$ & $168.16(4)$ \\
\hline $\mathrm{Au}(10)-\mathrm{Au}(4)-\mathrm{Au}(13)$ & $68.47(3)$ \\
\hline $\mathrm{Au}(11)-\mathrm{Au}(4)-\mathrm{Au}(13)$ & $126.05(4)$ \\
\hline $\mathrm{S}(4)-\mathrm{Au}(5)-\mathrm{Au}(1)$ & $153.20(15)$ \\
\hline $\mathrm{S}(4)-\mathrm{Au}(5)-\mathrm{Au}(2) \# 1$ & $142.21(14)$ \\
\hline
\end{tabular}




\begin{tabular}{|c|c|}
\hline $\mathrm{Au}(1)-\mathrm{Au}(5)-\mathrm{Au}(2) \# 1$ & $59.78(2)$ \\
\hline $\mathrm{S}(4)-\mathrm{Au}(5)-\mathrm{Au}(4)$ & $137.21(16)$ \\
\hline $\mathrm{Au}(1)-\mathrm{Au}(5)-\mathrm{Au}(4)$ & $57.90(2)$ \\
\hline $\mathrm{Au}(2) \# 1-\mathrm{Au}(5)-\mathrm{Au}(4)$ & $63.72(3)$ \\
\hline $\mathrm{S}(4)-\mathrm{Au}(5)-\mathrm{Au}(7)$ & $106.53(14)$ \\
\hline $\mathrm{Au}(1)-\mathrm{Au}(5)-\mathrm{Au}(7)$ & $58.21(2)$ \\
\hline $\mathrm{Au}(2) \# 1-\mathrm{Au}(5)-\mathrm{Au}(7)$ & $110.93(4)$ \\
\hline $\mathrm{Au}(4)-\mathrm{Au}(5)-\mathrm{Au}(7)$ & $59.67(3)$ \\
\hline $\mathrm{S}(4)-\mathrm{Au}(5)-\mathrm{Au}(3) \# 1$ & $113.99(16)$ \\
\hline $\mathrm{Au}(1)-\mathrm{Au}(5)-\mathrm{Au}(3) \# 1$ & $57.57(2)$ \\
\hline $\mathrm{Au}(2) \# 1-\mathrm{Au}(5)-\mathrm{Au}(3) \# 1$ & $60.30(3)$ \\
\hline $\mathrm{Au}(4)-\mathrm{Au}(5)-\mathrm{Au}(3) \# 1$ & $108.80(4)$ \\
\hline $\mathrm{Au}(7)-\mathrm{Au}(5)-\mathrm{Au}(3) \# 1$ & $105.53(3)$ \\
\hline$S(4)-A u(5)-A u(6)$ & $96.25(15)$ \\
\hline $\mathrm{Au}(1)-\mathrm{Au}(5)-\mathrm{Au}(6)$ & $57.12(2)$ \\
\hline $\mathrm{Au}(2) \# 1-\mathrm{Au}(5)-\mathrm{Au}(6)$ & $108.21(4)$ \\
\hline $\mathrm{Au}(4)-\mathrm{Au}(5)-\mathrm{Au}(6)$ & $104.88(3)$ \\
\hline $\mathrm{Au}(7)-\mathrm{Au}(5)-\mathrm{Au}(6)$ & $56.60(3)$ \\
\hline $\mathrm{Au}(3) \# 1-\mathrm{Au}(5)-\mathrm{Au}(6)$ & $59.20(3)$ \\
\hline $\mathrm{S}(4)-\mathrm{Au}(5)-\mathrm{Au}(9) \# 1$ & $80.04(15)$ \\
\hline $\mathrm{Au}(1)-\mathrm{Au}(5)-\mathrm{Au}(9) \# 1$ & $113.36(3)$ \\
\hline $\mathrm{Au}(2) \# 1-\mathrm{Au}(5)-\mathrm{Au}(9) \# 1$ & $63.31(3)$ \\
\hline $\mathrm{Au}(4)-\mathrm{Au}(5)-\mathrm{Au}(9) \# 1$ & $119.52(4)$ \\
\hline $\mathrm{Au}(7)-\mathrm{Au}(5)-\mathrm{Au}(9) \# 1$ & $171.15(4)$ \\
\hline $\mathrm{Au}(3) \# 1-\mathrm{Au}(5)-\mathrm{Au}(9) \# 1$ & $65.96(3)$ \\
\hline $\mathrm{Au}(6)-\mathrm{Au}(5)-\mathrm{Au}(9) \# 1$ & $117.55(3)$ \\
\hline $\mathrm{S}(4)-\mathrm{Au}(5)-\mathrm{Au}(11)$ & $95.16(16)$ \\
\hline $\mathrm{Au}(1)-\mathrm{Au}(5)-\mathrm{Au}(11)$ & $111.13(3)$ \\
\hline $\mathrm{Au}(2) \# 1-\mathrm{Au}(5)-\mathrm{Au}(11)$ & $64.55(3)$ \\
\hline $\mathrm{Au}(4)-\mathrm{Au}(5)-\mathrm{Au}(11)$ & $63.13(3)$ \\
\hline $\mathrm{Au}(7)-\mathrm{Au}(5)-\mathrm{Au}(11)$ & $115.15(3)$ \\
\hline $\mathrm{Au}(3) \# 1-\mathrm{Au}(5)-\mathrm{Au}(11)$ & $119.78(3)$ \\
\hline
\end{tabular}




\begin{tabular}{|c|c|}
\hline $\mathrm{Au}(6)-\mathrm{Au}(5)-\mathrm{Au}(11)$ & $167.59(4)$ \\
\hline $\mathrm{Au}(9) \# 1-\mathrm{Au}(5)-\mathrm{Au}(11)$ & $69.33(3)$ \\
\hline $\mathrm{S}(4)-\mathrm{Au}(5)-\mathrm{Au}(12)$ & $46.46(14)$ \\
\hline $\mathrm{Au}(1)-\mathrm{Au}(5)-\mathrm{Au}(12)$ & 109.94(3) \\
\hline $\mathrm{Au}(2) \# 1-\mathrm{Au}(5)-\mathrm{Au}(12)$ & $169.09(4)$ \\
\hline $\mathrm{Au}(4)-\mathrm{Au}(5)-\mathrm{Au}(12)$ & $115.21(3)$ \\
\hline $\mathrm{Au}(7)-\mathrm{Au}(5)-\mathrm{Au}(12)$ & $62.24(3)$ \\
\hline $\mathrm{Au}(3) \# 1-\mathrm{Au}(5)-\mathrm{Au}(12)$ & $111.99(3)$ \\
\hline $\mathrm{Au}(6)-\mathrm{Au}(5)-\mathrm{Au}(12)$ & $61.09(3)$ \\
\hline $\mathrm{Au}(9) \# 1-\mathrm{Au}(5)-\mathrm{Au}(12)$ & $122.27(4)$ \\
\hline $\mathrm{Au}(11)-\mathrm{Au}(5)-\mathrm{Au}(12)$ & $125.49(4)$ \\
\hline $\mathrm{S}(5) \# 1-\mathrm{Au}(6)-\mathrm{Au}(1)$ & $154.48(17)$ \\
\hline $\mathrm{S}(5) \# 1-\mathrm{Au}(6)-\mathrm{Au}(7)$ & $145.63(17)$ \\
\hline$A u(1)-A u(6)-A u(7)$ & $59.72(2)$ \\
\hline $\mathrm{S}(5) \# 1-\mathrm{Au}(6)-\mathrm{Au}(4) \# 1$ & $99.04(17)$ \\
\hline$A u(1)-A u(6)-A u(4) \# 1$ & $57.70(2)$ \\
\hline $\mathrm{Au}(7)-\mathrm{Au}(6)-\mathrm{Au}(4) \# 1$ & $110.33(4)$ \\
\hline $\mathrm{S}(5) \# 1-\mathrm{Au}(6)-\mathrm{Au}(3) \# 1$ & $101.69(16)$ \\
\hline $\mathrm{Au}(1)-\mathrm{Au}(6)-\mathrm{Au}(3) \# 1$ & $58.02(2)$ \\
\hline $\mathrm{Au}(7)-\mathrm{Au}(6)-\mathrm{Au}(3) \# 1$ & $109.16(3)$ \\
\hline $\mathrm{Au}(4) \# 1-\mathrm{Au}(6)-\mathrm{Au}(3) \# 1$ & $56.50(3)$ \\
\hline $\mathrm{S}(5) \# 1-\mathrm{Au}(6)-\mathrm{Au}(2)$ & $123.28(17)$ \\
\hline$A u(1)-A u(6)-A u(2)$ & $57.94(2)$ \\
\hline$A u(7)-A u(6)-A u(2)$ & $61.59(3)$ \\
\hline $\mathrm{Au}(4) \# 1-\mathrm{Au}(6)-\mathrm{Au}(2)$ & $61.40(3)$ \\
\hline $\mathrm{Au}(3) \# 1-\mathrm{Au}(6)-\mathrm{Au}(2)$ & $106.66(3)$ \\
\hline $\mathrm{S}(5) \# 1-\mathrm{Au}(6)-\mathrm{Au}(5)$ & $128.97(17)$ \\
\hline $\operatorname{Au}(1)-A u(6)-A u(5)$ & $57.62(2)$ \\
\hline $\mathrm{Au}(7)-\mathrm{Au}(6)-\mathrm{Au}(5)$ & $60.22(3)$ \\
\hline $\mathrm{Au}(4) \# 1-\mathrm{Au}(6)-\mathrm{Au}(5)$ & $105.30(3)$ \\
\hline $\mathrm{Au}(3) \# 1-\mathrm{Au}(6)-\mathrm{Au}(5)$ & $60.02(3)$ \\
\hline$A u(2)-A u(6)-A u(5)$ & $107.74(3)$ \\
\hline
\end{tabular}




\begin{tabular}{|c|c|}
\hline $\mathrm{S}(5) \# 1-\mathrm{Au}(6)-\mathrm{Au}(8)$ & $88.13(16)$ \\
\hline $\mathrm{Au}(1)-\mathrm{Au}(6)-\mathrm{Au}(8)$ & $111.52(3)$ \\
\hline $\mathrm{Au}(7)-\mathrm{Au}(6)-\mathrm{Au}(8)$ & $62.50(3)$ \\
\hline $\mathrm{Au}(4) \# 1-\mathrm{Au}(6)-\mathrm{Au}(8)$ & $118.80(4)$ \\
\hline $\mathrm{Au}(3) \# 1-\mathrm{Au}(6)-\mathrm{Au}(8)$ & $169.53(4)$ \\
\hline $\mathrm{Au}(2)-\mathrm{Au}(6)-\mathrm{Au}(8)$ & $64.28(3)$ \\
\hline$A u(5)-A u(6)-A u(8)$ & $116.29(4)$ \\
\hline $\mathrm{S}(5) \# 1-\mathrm{Au}(6)-\mathrm{Au}(12)$ & $90.42(17)$ \\
\hline $\mathrm{Au}(1)-\mathrm{Au}(6)-\mathrm{Au}(12)$ & $111.26(3)$ \\
\hline $\mathrm{Au}(7)-\mathrm{Au}(6)-\mathrm{Au}(12)$ & $63.78(3)$ \\
\hline $\mathrm{Au}(4) \# 1-\mathrm{Au}(6)-\mathrm{Au}(12)$ & $167.41(4)$ \\
\hline $\mathrm{Au}(3) \# 1-\mathrm{Au}(6)-\mathrm{Au}(12)$ & $113.57(4)$ \\
\hline $\mathrm{Au}(2)-\mathrm{Au}(6)-\mathrm{Au}(12)$ & $119.72(3)$ \\
\hline $\mathrm{Au}(5)-\mathrm{Au}(6)-\mathrm{Au}(12)$ & $62.13(3)$ \\
\hline$A u(8)-A u(6)-A u(12)$ & $69.49(3)$ \\
\hline $\mathrm{S}(5) \# 1-\mathrm{Au}(6)-\mathrm{Au}(10) \# 1$ & $45.60(16)$ \\
\hline $\mathrm{Au}(1)-\mathrm{Au}(6)-\mathrm{Au}(10) \# 1$ & 109.11(3) \\
\hline $\mathrm{Au}(7)-\mathrm{Au}(6)-\mathrm{Au}(10) \# 1$ & $168.77(4)$ \\
\hline $\mathrm{Au}(4) \# 1-\mathrm{Au}(6)-\mathrm{Au}(10) \# 1$ & $60.63(3)$ \\
\hline $\mathrm{Au}(3) \# 1-\mathrm{Au}(6)-\mathrm{Au}(10) \# 1$ & $60.79(3)$ \\
\hline $\mathrm{Au}(2)-\mathrm{Au}(6)-\mathrm{Au}(10) \# 1$ & $114.73(4)$ \\
\hline $\mathrm{Au}(5)-\mathrm{Au}(6)-\mathrm{Au}(10) \# 1$ & $113.98(3)$ \\
\hline $\mathrm{Au}(8)-\mathrm{Au}(6)-\mathrm{Au}(10) \# 1$ & $126.80(3)$ \\
\hline $\mathrm{Au}(12)-\mathrm{Au}(6)-\mathrm{Au}(10) \# 1$ & $123.52(4)$ \\
\hline$S(6)-A u(7)-A u(1)$ & $153.93(15)$ \\
\hline$S(6)-A u(7)-A u(6)$ & $144.65(14)$ \\
\hline$A u(1)-A u(7)-A u(6)$ & $59.56(2)$ \\
\hline $\mathrm{S}(6)-\mathrm{Au}(7)-\mathrm{Au}(4)$ & $103.68(14)$ \\
\hline $\mathrm{Au}(1)-\mathrm{Au}(7)-\mathrm{Au}(4)$ & $58.18(2)$ \\
\hline $\mathrm{Au}(6)-\mathrm{Au}(7)-\mathrm{Au}(4)$ & $110.46(3)$ \\
\hline$S(6)-A u(7)-A u(5)$ & $132.10(15)$ \\
\hline$A u(1)-A u(7)-A u(5)$ & $58.58(2)$ \\
\hline
\end{tabular}




\begin{tabular}{|c|c|}
\hline$A u(6)-A u(7)-A u(5)$ & $63.18(3)$ \\
\hline$A u(4)-A u(7)-A u(5)$ & $60.12(3)$ \\
\hline$S(6)-A u(7)-A u(2)$ & $117.66(15)$ \\
\hline$A u(1)-A u(7)-A u(2)$ & $58.00(2)$ \\
\hline$A u(6)-A u(7)-A u(2)$ & $61.90(3)$ \\
\hline$A u(4)-A u(7)-A u(2)$ & $105.21(4)$ \\
\hline$A u(5)-A u(7)-A u(2)$ & $110.20(3)$ \\
\hline$S(6)-A u(7)-A u(3)$ & $97.29(15)$ \\
\hline$A u(1)-A u(7)-A u(3)$ & $57.64(2)$ \\
\hline$A u(6)-A u(7)-A u(3)$ & $108.79(4)$ \\
\hline$A u(4)-A u(7)-A u(3)$ & $56.65(3)$ \\
\hline$A u(5)-A u(7)-A u(3)$ & $105.94(3)$ \\
\hline$A u(2)-A u(7)-A u(3)$ & $58.59(3)$ \\
\hline $\mathrm{S}(6)-\mathrm{Au}(7)-\mathrm{Au}(8)$ & $83.46(14)$ \\
\hline $\operatorname{Au}(1)-A u(7)-A u(8)$ & $112.62(3)$ \\
\hline$A u(6)-A u(7)-A u(8)$ & $63.99(3)$ \\
\hline$A u(4)-A u(7)-A u(8)$ & $169.93(4)$ \\
\hline$A u(5)-A u(7)-A u(8)$ & $120.16(4)$ \\
\hline$A u(2)-A u(7)-A u(8)$ & $64.90(3)$ \\
\hline$A u(3)-A u(7)-A u(8)$ & $115.94(4)$ \\
\hline$S(6)-A u(7)-A u(12)$ & $93.81(15)$ \\
\hline $\mathrm{Au}(1)-\mathrm{Au}(7)-\mathrm{Au}(12)$ & $110.71(3)$ \\
\hline$A u(6)-A u(7)-A u(12)$ & $63.25(3)$ \\
\hline $\mathrm{Au}(4)-\mathrm{Au}(7)-\mathrm{Au}(12)$ & $116.11(4)$ \\
\hline $\mathrm{Au}(5)-\mathrm{Au}(7)-\mathrm{Au}(12)$ & $62.81(3)$ \\
\hline $\mathrm{Au}(2)-\mathrm{Au}(7)-\mathrm{Au}(12)$ & $119.52(4)$ \\
\hline $\mathrm{Au}(3)-\mathrm{Au}(7)-\mathrm{Au}(12)$ & $167.99(4)$ \\
\hline $\mathrm{Au}(8)-\mathrm{Au}(7)-\mathrm{Au}(12)$ & $69.82(3)$ \\
\hline$S(6)-A u(7)-A u(13)$ & $44.88(14)$ \\
\hline $\mathrm{Au}(1)-\mathrm{Au}(7)-\mathrm{Au}(13)$ & $109.91(3)$ \\
\hline$A u(6)-A u(7)-A u(13)$ & $168.55(4)$ \\
\hline $\mathrm{Au}(4)-\mathrm{Au}(7)-\mathrm{Au}(13)$ & $63.07(3)$ \\
\hline
\end{tabular}




\begin{tabular}{|c|c|}
\hline $\mathrm{Au}(5)-\mathrm{Au}(7)-\mathrm{Au}(13)$ & $116.63(3)$ \\
\hline $\mathrm{Au}(2)-\mathrm{Au}(7)-\mathrm{Au}(13)$ & $109.51(4)$ \\
\hline $\mathrm{Au}(3)-\mathrm{Au}(7)-\mathrm{Au}(13)$ & $59.85(3)$ \\
\hline $\mathrm{Au}(8)-\mathrm{Au}(7)-\mathrm{Au}(13)$ & $120.76(3)$ \\
\hline $\mathrm{Au}(12)-\mathrm{Au}(7)-\mathrm{Au}(13)$ & $127.66(4)$ \\
\hline $\mathrm{S}(7)-\mathrm{Au}(8)-\mathrm{S}(1)$ & 173.91(19) \\
\hline$S(7)-A u(8)-A u(7)$ & $86.00(15)$ \\
\hline $\mathrm{S}(1)-\mathrm{Au}(8)-\mathrm{Au}(7)$ & $99.16(14)$ \\
\hline$S(7)-A u(8)-A u(6)$ & $91.89(14)$ \\
\hline $\mathrm{S}(1)-\mathrm{Au}(8)-\mathrm{Au}(6)$ & $93.79(14)$ \\
\hline $\operatorname{Au}(7)-A u(8)-A u(6)$ & $53.51(3)$ \\
\hline $\mathrm{S}(7)-\mathrm{Au}(8)-\mathrm{Au}(2)$ & $138.96(14)$ \\
\hline$S(1)-A u(8)-A u(2)$ & $47.00(13)$ \\
\hline $\mathrm{Au}(7)-\mathrm{Au}(8)-\mathrm{Au}(2)$ & $55.53(3)$ \\
\hline$A u(6)-A u(8)-A u(2)$ & $55.34(3)$ \\
\hline $\mathrm{S}(8)-\mathrm{Au}(9)-\mathrm{S}(2)$ & $173.1(2)$ \\
\hline $\mathrm{S}(8)-\mathrm{Au}(9)-\mathrm{Cd}(5) \# 1$ & $88.34(13)$ \\
\hline $\mathrm{S}(2)-\mathrm{Au}(9)-\mathrm{Cd}(5) \# 1$ & $95.01(14)$ \\
\hline $\mathrm{S}(8)-\mathrm{Au}(9)-\mathrm{Au}(5) \# 1$ & $88.34(13)$ \\
\hline $\mathrm{S}(2)-\mathrm{Au}(9)-\mathrm{Au}(5) \# 1$ & $95.01(14)$ \\
\hline $\mathrm{S}(8)-\mathrm{Au}(9)-\mathrm{Au}(2)$ & $92.69(15)$ \\
\hline $\mathrm{S}(2)-\mathrm{Au}(9)-\mathrm{Au}(2)$ & $94.11(15)$ \\
\hline $\mathrm{Au}(5) \# 1-\mathrm{Au}(9)-\mathrm{Au}(2)$ & $53.66(3)$ \\
\hline $\mathrm{S}(8)-\mathrm{Au}(9)-\mathrm{Au}(3)$ & $139.87(14)$ \\
\hline$S(2)-A u(9)-A u(3)$ & $45.72(13)$ \\
\hline $\mathrm{Au}(5) \# 1-\mathrm{Au}(9)-\mathrm{Au}(3)$ & $55.23(2)$ \\
\hline$A u(2)-A u(9)-A u(3)$ & $53.74(2)$ \\
\hline $\mathrm{S}(5)-\mathrm{Au}(10)-\mathrm{S}(9)$ & $172.1(2)$ \\
\hline $\mathrm{S}(5)-\mathrm{Au}(10)-\mathrm{Au}(4)$ & $95.42(16)$ \\
\hline$S(9)-A u(10)-A u(4)$ & $91.00(16)$ \\
\hline$S(5)-A u(10)-A u(3)$ & $97.88(14)$ \\
\hline$S(9)-A u(10)-A u(3)$ & $89.59(14)$ \\
\hline
\end{tabular}




\begin{tabular}{|c|c|}
\hline $\mathrm{Au}(4)-\mathrm{Au}(10)-\mathrm{Au}(3)$ & $52.92(3)$ \\
\hline$S(5)-A u(10)-C d(6) \# 1$ & $47.13(14)$ \\
\hline$S(9)-A u(10)-C d(6) \# 1$ & $140.68(15)$ \\
\hline $\mathrm{S}(5)-\mathrm{Au}(10)-\mathrm{Au}(6) \# 1$ & $47.13(14)$ \\
\hline$S(9)-A u(10)-A u(6) \# 1$ & $140.68(15)$ \\
\hline $\mathrm{Au}(4)-\mathrm{Au}(10)-\mathrm{Au}(6) \# 1$ & $55.15(3)$ \\
\hline $\mathrm{Au}(3)-\mathrm{Au}(10)-\mathrm{Au}(6) \# 1$ & $55.21(2)$ \\
\hline $\mathrm{S}(8) \# 1-\mathrm{Au}(11)-\mathrm{S}(3)$ & $171.84(19)$ \\
\hline $\mathrm{S}(8) \# 1-\mathrm{Au}(11)-\mathrm{Au}(5)$ & $87.56(14)$ \\
\hline$S(3)-A u(11)-A u(5)$ & $100.59(13)$ \\
\hline $\mathrm{S}(8) \# 1-\mathrm{Au}(11)-\mathrm{Au}(4)$ & $139.97(14)$ \\
\hline $\mathrm{S}(3)-\mathrm{Au}(11)-\mathrm{Au}(4)$ & $47.83(13)$ \\
\hline $\mathrm{Au}(5)-\mathrm{Au}(11)-\mathrm{Au}(4)$ & $54.94(2)$ \\
\hline $\mathrm{S}(8) \# 1-\mathrm{Au}(11)-\mathrm{Au}(2) \# 1$ & $90.92(14)$ \\
\hline $\mathrm{S}(3)-\mathrm{Au}(11)-\mathrm{Au}(2) \# 1$ & $94.13(15)$ \\
\hline $\mathrm{Au}(5)-\mathrm{Au}(11)-\mathrm{Au}(2) \# 1$ & $52.54(2)$ \\
\hline $\mathrm{Au}(4)-\mathrm{Au}(11)-\mathrm{Au}(2) \# 1$ & $56.64(3)$ \\
\hline $\mathrm{S}(8) \# 1-\mathrm{Au}(11)-\mathrm{Cd}(2) \# 1$ & $90.92(14)$ \\
\hline $\mathrm{S}(3)-\mathrm{Au}(11)-\mathrm{Cd}(2) \# 1$ & $94.13(15)$ \\
\hline $\mathrm{S}(7)-\mathrm{Au}(12)-\mathrm{S}(4)$ & $173.3(2)$ \\
\hline$S(7)-A u(12)-A u(6)$ & $91.80(17)$ \\
\hline$S(4)-A u(12)-A u(6)$ & $94.15(17)$ \\
\hline$S(7)-A u(12)-A u(7)$ & $84.61(15)$ \\
\hline $\mathrm{S}(4)-\mathrm{Au}(12)-\mathrm{Au}(7)$ & $101.37(16)$ \\
\hline$A u(6)-A u(12)-A u(7)$ & $52.96(3)$ \\
\hline$S(7)-A u(12)-A u(5)$ & $138.10(16)$ \\
\hline$S(4)-A u(12)-A u(5)$ & $48.44(16)$ \\
\hline $\mathrm{Au}(6)-\mathrm{Au}(12)-\mathrm{Au}(5)$ & $56.77(3)$ \\
\hline $\mathrm{Au}(7)-\mathrm{Au}(12)-\mathrm{Au}(5)$ & $54.95(3)$ \\
\hline$S(9)-A u(13)-S(6)$ & $174.50(19)$ \\
\hline $\mathrm{S}(9)-\mathrm{Au}(13)-\mathrm{Au}(3)$ & $90.46(14)$ \\
\hline$S(6)-A u(13)-A u(3)$ & $94.83(14)$ \\
\hline
\end{tabular}




\begin{tabular}{|c|c|}
\hline $\mathrm{S}(9)-\mathrm{Au}(13)-\mathrm{Au}(4)$ & $88.77(14)$ \\
\hline$S(6)-A u(13)-A u(4)$ & $95.68(13)$ \\
\hline $\mathrm{Au}(3)-\mathrm{Au}(13)-\mathrm{Au}(4)$ & $52.06(3)$ \\
\hline $\mathrm{S}(9)-\mathrm{Au}(13)-\mathrm{Au}(7)$ & $138.90(14)$ \\
\hline$S(6)-A u(13)-A u(7)$ & $46.41(13)$ \\
\hline $\mathrm{Au}(3)-\mathrm{Au}(13)-\mathrm{Au}(7)$ & $55.65(3)$ \\
\hline $\mathrm{Au}(4)-\mathrm{Au}(13)-\mathrm{Au}(7)$ & $53.08(2)$ \\
\hline$C(1 \mathrm{~A})-\mathrm{S}(1)-\mathrm{Au}(8)$ & $104.6(8)$ \\
\hline $\mathrm{C}(1 \mathrm{~A})-\mathrm{S}(1)-\mathrm{Au}(2)$ & $111.9(8)$ \\
\hline $\mathrm{Au}(8)-\mathrm{S}(1)-\mathrm{Au}(2)$ & $88.2(2)$ \\
\hline $\mathrm{C}(2 \mathrm{~A})-\mathrm{C}(1 \mathrm{~A})-\mathrm{S}(1)$ & $120.2(16)$ \\
\hline$C(1 \mathrm{~A})-\mathrm{C}(2 \mathrm{~A})-\mathrm{C}(3 \mathrm{~A})$ & $103(2)$ \\
\hline$C(8 A)-C(3 A)-C(4 A)$ & $127(3)$ \\
\hline$C(8 A)-C(3 A)-C(2 A)$ & $115(3)$ \\
\hline$C(4 A)-C(3 A)-C(2 A)$ & $118(3)$ \\
\hline$C(3 A)-C(4 A)-C(5 A)$ & $110(3)$ \\
\hline$C(6 A)-C(5 A)-C(4 A)$ & $123(4)$ \\
\hline$C(7 A)-C(6 A)-C(5 A)$ & 121(3) \\
\hline$C(6 A)-C(7 A)-C(8 A)$ & 113(3) \\
\hline$C(3 A)-C(8 A)-C(7 A)$ & $123(3)$ \\
\hline C(1B)-S(2)-Au(9) & 104.3(9) \\
\hline$C(1 B)-S(2)-A u(3)$ & 101.9(9) \\
\hline $\mathrm{Au}(9)-\mathrm{S}(2)-\mathrm{Au}(3)$ & $90.11(18)$ \\
\hline C(2B)-C(1B)-S(2) & $120(2)$ \\
\hline $\mathrm{C}(3 \mathrm{~B})-\mathrm{C}(2 \mathrm{~B})-\mathrm{C}(1 \mathrm{~B})$ & $107.9(18)$ \\
\hline $\mathrm{C}(4 \mathrm{~B})-\mathrm{C}(3 \mathrm{~B})-\mathrm{C}(8 \mathrm{~B})$ & 120.0 \\
\hline $\mathrm{C}(4 \mathrm{~B})-\mathrm{C}(3 \mathrm{~B})-\mathrm{C}(2 \mathrm{~B})$ & $121.2(11)$ \\
\hline $\mathrm{C}(8 \mathrm{~B})-\mathrm{C}(3 \mathrm{~B})-\mathrm{C}(2 \mathrm{~B})$ & $118.8(11)$ \\
\hline $\mathrm{C}(3 \mathrm{~B})-\mathrm{C}(4 \mathrm{~B})-\mathrm{C}(5 \mathrm{~B})$ & 120.0 \\
\hline$C(6 B)-C(5 B)-C(4 B)$ & 120.0 \\
\hline$C(5 B)-C(6 B)-C(7 B)$ & 120.0 \\
\hline $\mathrm{C}(8 \mathrm{~B})-\mathrm{C}(7 \mathrm{~B})-\mathrm{C}(6 \mathrm{~B})$ & 120.0 \\
\hline
\end{tabular}




\begin{tabular}{|c|c|}
\hline $\mathrm{C}(7 \mathrm{~B})-\mathrm{C}(8 \mathrm{~B})-\mathrm{C}(3 \mathrm{~B})$ & 120.0 \\
\hline $\mathrm{C}(1 \mathrm{C})-\mathrm{S}(3)-\mathrm{Au}(11)$ & $106.4(8)$ \\
\hline$C(1 C)-S(3)-A u(4)$ & 109.1(8) \\
\hline $\mathrm{Au}(11)-\mathrm{S}(3)-\mathrm{Au}(4)$ & $85.84(17)$ \\
\hline$C(2 C)-C(1 C)-S(3)$ & $107.4(17)$ \\
\hline$C(3 C)-C(2 C)-C(1 C)$ & 113(2) \\
\hline $\mathrm{C}(8 \mathrm{C})-\mathrm{C}(3 \mathrm{C})-\mathrm{C}(4 \mathrm{C})$ & $117(3)$ \\
\hline $\mathrm{C}(8 \mathrm{C})-\mathrm{C}(3 \mathrm{C})-\mathrm{C}(2 \mathrm{C})$ & $121(2)$ \\
\hline$C(4 C)-C(3 C)-C(2 C)$ & $121(3)$ \\
\hline$C(5 C)-C(4 C)-C(3 C)$ & 123(4) \\
\hline$C(6 C)-C(5 C)-C(4 C)$ & $119(4)$ \\
\hline$C(5 C)-C(6 C)-C(7 C)$ & $124(4)$ \\
\hline$C(8 C)-C(7 C)-C(6 C)$ & $120(3)$ \\
\hline$C(7 C)-C(8 C)-C(3 C)$ & $115(3)$ \\
\hline$C(1 D)-S(4)-A u(12)$ & $103.2(13)$ \\
\hline$C(1 D)-S(4)-A u(5)$ & $105.3(10)$ \\
\hline$A u(12)-S(4)-A u(5)$ & $85.1(2)$ \\
\hline$C(2 D)-C(1 D)-S(4)$ & $115(2)$ \\
\hline $\mathrm{C}(3 \mathrm{D})-\mathrm{C}(2 \mathrm{D})-\mathrm{C}(1 \mathrm{D})$ & $111.3(17)$ \\
\hline$C(4 D)-C(3 D)-C(8 D)$ & 120.0 \\
\hline$C(4 D)-C(3 D)-C(2 D)$ & $115(2)$ \\
\hline $\mathrm{C}(8 \mathrm{D})-\mathrm{C}(3 \mathrm{D})-\mathrm{C}(2 \mathrm{D})$ & $125(2)$ \\
\hline$C(3 D)-C(4 D)-C(5 D)$ & 120.0 \\
\hline$C(6 D)-C(5 D)-C(4 D)$ & 120.0 \\
\hline$C(5 D)-C(6 D)-C(7 D)$ & 120.0 \\
\hline$C(6 D)-C(7 D)-C(8 D)$ & 120.0 \\
\hline C(7D)-C(8D)-C(3D) & 120.0 \\
\hline$C(1 E)-S(5)-A u(10)$ & $106.7(13)$ \\
\hline$C(1 E)-S(5)-C d(6) \# 1$ & $100.3(14)$ \\
\hline $\mathrm{Au}(10)-\mathrm{S}(5)-\mathrm{Cd}(6) \# 1$ & $87.3(2)$ \\
\hline$C(1 E)-S(5)-A u(6) \# 1$ & $100.3(14)$ \\
\hline $\mathrm{Au}(10)-\mathrm{S}(5)-\mathrm{Au}(6) \# 1$ & $87.3(2)$ \\
\hline
\end{tabular}




\begin{tabular}{|c|c|}
\hline$C(2 E)-C(1 E)-S(5)$ & 111(2) \\
\hline$C(3 E)-C(2 E)-C(1 E)$ & $107(3)$ \\
\hline$C(4 E)-C(3 E)-C(8 E)$ & $113(3)$ \\
\hline$C(4 E)-C(3 E)-C(2 E)$ & $108(3)$ \\
\hline$C(8 E)-C(3 E)-C(2 E)$ & $140(3)$ \\
\hline$C(3 E)-C(4 E)-C(5 E)$ & $135(3)$ \\
\hline$C(4 E)-C(5 E)-C(6 E)$ & $110(4)$ \\
\hline$C(5 E)-C(6 E)-C(7 E)$ & $120(4)$ \\
\hline $\mathrm{C}(6 \mathrm{E})-\mathrm{C}(7 \mathrm{E})-\mathrm{C}(8 \mathrm{E})$ & $124(3)$ \\
\hline$C(3 E)-C(8 E)-C(7 E)$ & $118(3)$ \\
\hline $\mathrm{C}(1 \mathrm{~F})-\mathrm{S}(6)-\mathrm{Au}(13)$ & $105.2(8)$ \\
\hline$C(1 F)-S(6)-A u(7)$ & 109.7(7) \\
\hline $\mathrm{Au}(13)-\mathrm{S}(6)-\mathrm{Au}(7)$ & $88.71(19)$ \\
\hline$C(2 F)-C(1 F)-S(6)$ & $111.8(16)$ \\
\hline$C(1 F)-C(2 F)-C(3 F)$ & $113.2(18)$ \\
\hline $\mathrm{C}(4 \mathrm{~F})-\mathrm{C}(3 \mathrm{~F})-\mathrm{C}(8 \mathrm{~F})$ & 120.0 \\
\hline$C(4 F)-C(3 F)-C(2 F)$ & $122.6(15)$ \\
\hline$C(8 F)-C(3 F)-C(2 F)$ & $117.3(15)$ \\
\hline$C(3 F)-C(4 F)-C(5 F)$ & 120.0 \\
\hline$C(6 F)-C(5 F)-C(4 F)$ & 120.0 \\
\hline$C(5 F)-C(6 F)-C(7 F)$ & 120.0 \\
\hline$C(6 F)-C(7 F)-C(8 F)$ & 120.0 \\
\hline $\mathrm{C}(7 \mathrm{~F})-\mathrm{C}(8 \mathrm{~F})-\mathrm{C}(3 \mathrm{~F})$ & 120.0 \\
\hline $\mathrm{C}(1 \mathrm{G})-\mathrm{S}(7)-\mathrm{Au}(8)$ & 104.3(8) \\
\hline$C(1 G)-S(7)-A u(12)$ & $104.0(8)$ \\
\hline $\mathrm{Au}(8)-\mathrm{S}(7)-\mathrm{Au}(12)$ & $102.7(2)$ \\
\hline$C(2 G)-C(1 G)-S(7)$ & $111.7(19)$ \\
\hline$C(1 G)-C(2 G)-C(3 G)$ & $110(2)$ \\
\hline $\mathrm{C}(8 \mathrm{G})-\mathrm{C}(3 \mathrm{G})-\mathrm{C}(4 \mathrm{G})$ & $123(3)$ \\
\hline $\mathrm{C}(8 \mathrm{G})-\mathrm{C}(3 \mathrm{G})-\mathrm{C}(2 \mathrm{G})$ & $110(2)$ \\
\hline$C(4 G)-C(3 G)-C(2 G)$ & $127(2)$ \\
\hline$C(5 G)-C(4 G)-C(3 G)$ & 111(3) \\
\hline
\end{tabular}




\begin{tabular}{|c|c|}
\hline$C(4 G)-C(5 G)-C(6 G)$ & 131(3) \\
\hline$C(7 G)-C(6 G)-C(5 G)$ & $106(3)$ \\
\hline $\mathrm{C}(6 \mathrm{G})-\mathrm{C}(7 \mathrm{G})-\mathrm{C}(8 \mathrm{G})$ & $133(4)$ \\
\hline $\mathrm{C}(3 \mathrm{G})-\mathrm{C}(8 \mathrm{G})-\mathrm{C}(7 \mathrm{G})$ & 111(3) \\
\hline $\mathrm{C}(1 \mathrm{H})-\mathrm{S}(8)-\mathrm{Au}(11) \# 1$ & $102.2(8)$ \\
\hline $\mathrm{C}(1 \mathrm{H})-\mathrm{S}(8)-\mathrm{Au}(9)$ & 106.7(9) \\
\hline $\mathrm{Au}(11) \# 1-\mathrm{S}(8)-\mathrm{Au}(9)$ & $101.4(2)$ \\
\hline $\mathrm{C}(2 \mathrm{H})-\mathrm{C}(1 \mathrm{H})-\mathrm{S}(8)$ & 109.2(16) \\
\hline $\mathrm{C}(3 \mathrm{H})-\mathrm{C}(2 \mathrm{H})-\mathrm{C}(1 \mathrm{H})$ & 107.1(17) \\
\hline $\mathrm{C}(8 \mathrm{H})-\mathrm{C}(3 \mathrm{H})-\mathrm{C}(4 \mathrm{H})$ & $119(2)$ \\
\hline $\mathrm{C}(8 \mathrm{H})-\mathrm{C}(3 \mathrm{H})-\mathrm{C}(2 \mathrm{H})$ & $120(2)$ \\
\hline $\mathrm{C}(4 \mathrm{H})-\mathrm{C}(3 \mathrm{H})-\mathrm{C}(2 \mathrm{H})$ & $120.5(18)$ \\
\hline $\mathrm{C}(5 \mathrm{H})-\mathrm{C}(4 \mathrm{H})-\mathrm{C}(3 \mathrm{H})$ & $122(3)$ \\
\hline $\mathrm{C}(4 \mathrm{H})-\mathrm{C}(5 \mathrm{H})-\mathrm{C}(6 \mathrm{H})$ & $118(3)$ \\
\hline $\mathrm{C}(7 \mathrm{H})-\mathrm{C}(6 \mathrm{H})-\mathrm{C}(5 \mathrm{H})$ & $119(3)$ \\
\hline $\mathrm{C}(6 \mathrm{H})-\mathrm{C}(7 \mathrm{H})-\mathrm{C}(8 \mathrm{H})$ & 124(3) \\
\hline $\mathrm{C}(3 \mathrm{H})-\mathrm{C}(8 \mathrm{H})-\mathrm{C}(7 \mathrm{H})$ & $117(2)$ \\
\hline$C(1 \mathrm{I})-\mathrm{S}(9)-\mathrm{Au}(13)$ & 108.2(9) \\
\hline$C(1 \mathrm{I})-\mathrm{S}(9)-\mathrm{Au}(10)$ & $106.6(9)$ \\
\hline $\mathrm{Au}(13)-\mathrm{S}(9)-\mathrm{Au}(10)$ & $101.6(2)$ \\
\hline $\mathrm{C}(2 \mathrm{I})-\mathrm{C}(1 \mathrm{I})-\mathrm{S}(9)$ & $117.8(19)$ \\
\hline $\mathrm{C}(3 \mathrm{I})-\mathrm{C}(2 \mathrm{I})-\mathrm{C}(1 \mathrm{I})$ & $112.8(19)$ \\
\hline $\mathrm{C}(4 \mathrm{I})-\mathrm{C}(3 \mathrm{I})-\mathrm{C}(8 \mathrm{I})$ & $114(3)$ \\
\hline $\mathrm{C}(4 \mathrm{I})-\mathrm{C}(3 \mathrm{I})-\mathrm{C}(2 \mathrm{I})$ & $126(3)$ \\
\hline $\mathrm{C}(8 \mathrm{I})-\mathrm{C}(3 \mathrm{I})-\mathrm{C}(2 \mathrm{I})$ & $119(3)$ \\
\hline$C(3 \mathrm{I})-\mathrm{C}(4 \mathrm{I})-\mathrm{C}(5 \mathrm{I})$ & $122(3)$ \\
\hline$C(6 \mathrm{I})-\mathrm{C}(5 \mathrm{I})-\mathrm{C}(4 \mathrm{I})$ & $123(2)$ \\
\hline$C(5 \mathrm{I})-\mathrm{C}(6 \mathrm{I})-\mathrm{C}(7 \mathrm{I})$ & $122(3)$ \\
\hline$C(8 I)-C(7 I)-C(6 I)$ & $114(3)$ \\
\hline$C(7 \mathrm{I})-\mathrm{C}(8 \mathrm{I})-\mathrm{C}(3 \mathrm{I})$ & $126(3)$ \\
\hline $\mathrm{C}(7)-\mathrm{C}(2)-\mathrm{C}(3)$ & 121(3) \\
\hline$C(7)-C(2)-C(1)$ & $139(4)$ \\
\hline
\end{tabular}


$\mathrm{C}(3)-\mathrm{C}(2)-\mathrm{C}(1)$

$96(3)$

$\mathrm{C}(2)-\mathrm{C}(3)-\mathrm{C}(4)$

$\mathrm{C}(5)-\mathrm{C}(4)-\mathrm{C}(3)$

$\mathrm{C}(4)-\mathrm{C}(5)-\mathrm{C}(6)$

$\mathrm{C}(5)-\mathrm{C}(6)-\mathrm{C}(7)$

$\mathrm{C}(2)-\mathrm{C}(7)-\mathrm{C}(6)$
$110(3)$

130(4)

$114(3)$

$118(3)$

124(3)

Symmetry transformations used to generate equivalent atoms:

$\# 1-\mathrm{x},-\mathrm{y},-\mathrm{z}+2$ 
Table S6. Anisotropic displacement parameters $\left(\AA^{2} \mathrm{x} 10^{3}\right)$ for $\mathrm{Au}_{24} \mathrm{Cd}(\mathrm{PET})_{18}$. The anisotropic displacement factor exponent takes the form: $-2 p^{2}\left[h^{2} a^{* 2} U^{11}+\ldots+2 h k a^{*} b^{*} U^{12}\right]$

\begin{tabular}{|c|c|c|c|c|c|c|}
\hline & $\mathrm{U}^{11}$ & $\mathrm{U}^{22}$ & $\mathrm{U}^{33}$ & $\mathrm{U}^{23}$ & $\mathrm{U}^{13}$ & $\mathrm{U}^{12}$ \\
\hline $\mathrm{Au}(1)$ & $15(1)$ & $9(1)$ & $16(1)$ & $-5(1)$ & $-8(1)$ & 1(1) \\
\hline $\mathrm{Au}(2)$ & $24(1)$ & 12(1) & 21(1) & $-7(1)$ & $-11(1)$ & 2(1) \\
\hline $\mathrm{Au}(3)$ & $20(1)$ & $20(1)$ & $23(1)$ & $-10(1)$ & $-12(1)$ & $1(1)$ \\
\hline $\mathrm{Au}(4)$ & $16(1)$ & $24(1)$ & $24(1)$ & $-13(1)$ & $-7(1)$ & $3(1)$ \\
\hline $\mathrm{Au}(5)$ & $26(1)$ & $14(1)$ & $16(1)$ & $-5(1)$ & $-8(1)$ & $0(1)$ \\
\hline $\mathrm{Au}(6)$ & $27(1)$ & $14(1)$ & $27(1)$ & $-6(1)$ & $-18(1)$ & $4(1)$ \\
\hline $\mathrm{Au}(7)$ & 21(1) & $14(1)$ & $23(1)$ & $-5(1)$ & $-7(1)$ & $-5(1)$ \\
\hline $\mathrm{Cd}(2)$ & $24(1)$ & $12(1)$ & 21(1) & $-7(1)$ & $-11(1)$ & $2(1)$ \\
\hline $\mathrm{Cd}(3)$ & $20(1)$ & 20(1) & $23(1)$ & $-10(1)$ & $-12(1)$ & $1(1)$ \\
\hline $\mathrm{Cd}(4)$ & $16(1)$ & $24(1)$ & $24(1)$ & $-13(1)$ & $-7(1)$ & $3(1)$ \\
\hline $\mathrm{Cd}(5)$ & $26(1)$ & $14(1)$ & $16(1)$ & $-5(1)$ & $-8(1)$ & $0(1)$ \\
\hline $\operatorname{Cd}(6)$ & $27(1)$ & $14(1)$ & $27(1)$ & $-6(1)$ & $-18(1)$ & $4(1)$ \\
\hline $\mathrm{Cd}(7)$ & 21(1) & $14(1)$ & $23(1)$ & $-5(1)$ & $-7(1)$ & $-5(1)$ \\
\hline $\mathrm{Au}(8)$ & $49(1)$ & $12(1)$ & $28(1)$ & $-6(1)$ & $-21(1)$ & $3(1)$ \\
\hline $\mathrm{Au}(9)$ & 21(1) & $22(1)$ & $26(1)$ & $-14(1)$ & $-9(1)$ & $0(1)$ \\
\hline $\operatorname{Au}(10)$ & $19(1)$ & $26(1)$ & $28(1)$ & $-11(1)$ & $-12(1)$ & $2(1)$ \\
\hline $\operatorname{Au}(11)$ & 21(1) & $23(1)$ & $34(1)$ & $-18(1)$ & $-8(1)$ & $0(1)$ \\
\hline $\operatorname{Au}(12)$ & $35(1)$ & $18(1)$ & $26(1)$ & $-7(1)$ & $-14(1)$ & $4(1)$ \\
\hline $\operatorname{Au}(13)$ & $22(1)$ & $25(1)$ & $34(1)$ & $-17(1)$ & $-6(1)$ & $-4(1)$ \\
\hline $\mathrm{S}(1)$ & $36(3)$ & $18(3)$ & $39(4)$ & $-16(3)$ & $-20(3)$ & $9(2)$ \\
\hline$C(1 \mathrm{~A})$ & $19(10)$ & $31(13)$ & $33(14)$ & $-25(12)$ & $0(10)$ & $-6(9)$ \\
\hline $\mathrm{C}(2 \mathrm{~A})$ & $23(12)$ & $64(19)$ & $48(17)$ & $-43(16)$ & $10(12)$ & $-16(12)$ \\
\hline$C(3 A)$ & $58(15)$ & $53(14)$ & $89(18)$ & $-53(14)$ & $-12(13)$ & $-21(11)$ \\
\hline$C(4 A)$ & $44(13)$ & $82(16)$ & $64(16)$ & $-55(14)$ & $-13(12)$ & $-8(11)$ \\
\hline$C(5 \mathrm{~A})$ & $100(20)$ & $140(20)$ & $110(20)$ & $-75(18)$ & $-34(16)$ & $2(16)$ \\
\hline$C(6 A)$ & $43(13)$ & $64(15)$ & $88(18)$ & $-66(14)$ & $16(12)$ & $-21(11)$ \\
\hline $\mathrm{C}(7 \mathrm{~A})$ & $47(11)$ & $53(11)$ & $73(12)$ & $-32(9)$ & $-5(8)$ & $-17(8)$ \\
\hline
\end{tabular}




\begin{tabular}{|c|c|c|c|c|c|c|}
\hline $\mathrm{C}(8 \mathrm{~A})$ & $84(18)$ & $85(18)$ & $110(20)$ & $-64(15)$ & $-51(15)$ & $6(13)$ \\
\hline$S(2)$ & $20(3)$ & $47(4)$ & $30(3)$ & $-26(3)$ & $-9(2)$ & $-1(2)$ \\
\hline$C(1 B)$ & $29(10)$ & $78(13)$ & $35(11)$ & $-22(10)$ & $-12(9)$ & $3(10)$ \\
\hline $\mathrm{C}(2 \mathrm{~B})$ & $75(12)$ & $94(13)$ & $74(13)$ & $-26(11)$ & $-25(10)$ & $-4(11)$ \\
\hline$C(3 B)$ & $75(12)$ & 101(13) & $98(13)$ & $-17(11)$ & $-48(11)$ & $-5(11)$ \\
\hline $\mathrm{C}(4 \mathrm{~B})$ & $86(17)$ & $103(18)$ & $112(19)$ & $-12(16)$ & $-28(16)$ & $-10(15)$ \\
\hline$C(5 B)$ & $122(19)$ & $110(20)$ & $110(20)$ & $-17(18)$ & $-40(17)$ & $6(17)$ \\
\hline$C(6 B)$ & $130(20)$ & $100(20)$ & $110(20)$ & $-25(17)$ & $-43(18)$ & $-7(18)$ \\
\hline$C(7 B)$ & $123(19)$ & $120(20)$ & $110(20)$ & $-4(17)$ & $-71(16)$ & $-12(17)$ \\
\hline $\mathrm{C}(8 \mathrm{~B})$ & $116(17)$ & $119(18)$ & $97(17)$ & $4(15)$ & $-88(14)$ & $-7(15)$ \\
\hline $\mathrm{S}(3)$ & $17(3)$ & $40(4)$ & $37(4)$ & $-26(3)$ & $-9(2)$ & $9(2)$ \\
\hline$C(1 C)$ & $29(9)$ & $41(9)$ & $42(10)$ & $-23(8)$ & $-8(7)$ & $-12(7)$ \\
\hline$C(2 C)$ & $11(7)$ & $33(9)$ & $22(9)$ & $-7(7)$ & $0(6)$ & $-6(6)$ \\
\hline$C(3 C)$ & $23(6)$ & $36(7)$ & $31(7)$ & $-13(5)$ & $-8(5)$ & $-7(4)$ \\
\hline$C(4 C)$ & $120(20)$ & $110(20)$ & $100(20)$ & $-77(18)$ & $-11(15)$ & $-1(16)$ \\
\hline$C(5 C)$ & $33(13)$ & $100(18)$ & $79(18)$ & $-47(15)$ & $3(12)$ & $-36(12)$ \\
\hline$C(6 C)$ & 73(17) & $76(17)$ & $74(18)$ & $-39(14)$ & $-18(14)$ & $-18(13)$ \\
\hline$C(7 C)$ & $28(11)$ & $43(13)$ & $46(14)$ & $-30(11)$ & $10(10)$ & $-12(9)$ \\
\hline$C(8 C)$ & $86(16)$ & $64(16)$ & $61(15)$ & $-27(12)$ & $-64(13)$ & $25(12)$ \\
\hline$S(4)$ & $56(4)$ & $23(3)$ & $26(3)$ & $-10(3)$ & $-21(3)$ & $6(3)$ \\
\hline$C(1 D)$ & $84(12)$ & $60(12)$ & $61(12)$ & $-2(10)$ & $-18(10)$ & $20(10)$ \\
\hline$C(2 D)$ & $105(13)$ & $89(13)$ & $78(13)$ & $7(11)$ & $-47(11)$ & $17(12)$ \\
\hline$C(3 D)$ & $129(16)$ & $98(16)$ & $122(17)$ & $47(14)$ & $-35(15)$ & $6(15)$ \\
\hline$C(4 D)$ & $107(18)$ & $94(18)$ & $140(20)$ & $39(17)$ & $-25(18)$ & $-33(16)$ \\
\hline$C(5 D)$ & $84(18)$ & $70(18)$ & $120(20)$ & $25(17)$ & $-20(17)$ & $-31(15)$ \\
\hline$C(6 D)$ & $130(20)$ & $69(18)$ & $110(20)$ & $29(17)$ & $-29(18)$ & $8(16)$ \\
\hline$C(7 D)$ & $120(20)$ & $77(18)$ & $120(20)$ & $25(17)$ & $-22(19)$ & $-5(17)$ \\
\hline$C(8 D)$ & $130(20)$ & $94(18)$ & $120(20)$ & $44(17)$ & $-32(18)$ & $-11(17)$ \\
\hline$S(5)$ & $34(3)$ & $30(3)$ & $40(4)$ & $-8(3)$ & $-22(3)$ & $13(3)$ \\
\hline$C(1 E)$ & $84(19)$ & $82(19)$ & $110(20)$ & $-58(16)$ & $-17(15)$ & $22(15)$ \\
\hline$C(2 E)$ & $100(20)$ & $100(20)$ & $90(20)$ & $-71(17)$ & $13(14)$ & $-2(15)$ \\
\hline$C(3 E)$ & $31(12)$ & $52(14)$ & $57(15)$ & $-33(12)$ & $8(10)$ & $-29(10)$ \\
\hline
\end{tabular}




\begin{tabular}{|c|c|c|c|c|c|c|}
\hline$C(4 E)$ & $93(18)$ & 81(19) & $100(20)$ & $-22(15)$ & $-63(15)$ & $-36(14)$ \\
\hline$C(5 E)$ & $120(30)$ & $130(30)$ & $150(30)$ & $-60(19)$ & $-50(19)$ & $-15(17)$ \\
\hline$C(6 E)$ & $115(19)$ & $110(20)$ & $120(20)$ & $-58(17)$ & $-34(16)$ & $58(16)$ \\
\hline$C(7 E)$ & 101(17) & $93(18)$ & $75(18)$ & $-54(15)$ & $-36(14)$ & $0(13)$ \\
\hline$C(8 E)$ & $56(14)$ & $62(15)$ & $60(16)$ & $-29(13)$ & $-8(12)$ & $4(11)$ \\
\hline$S(6)$ & $27(3)$ & $13(3)$ & $32(3)$ & $-7(2)$ & $-6(2)$ & $-5(2)$ \\
\hline $\mathrm{C}(1 \mathrm{~F})$ & 41(9) & $27(9)$ & $31(9)$ & $-8(7)$ & $-21(7)$ & $0(7)$ \\
\hline $\mathrm{C}(2 \mathrm{~F})$ & $26(8)$ & $33(9)$ & $27(9)$ & $6(7)$ & $-15(7)$ & $2(7)$ \\
\hline $\mathrm{C}(3 \mathrm{~F})$ & $43(9)$ & $44(10)$ & $39(10)$ & $-14(8)$ & $-22(8)$ & $14(8)$ \\
\hline $\mathrm{C}(4 \mathrm{~F})$ & $37(14)$ & $48(18)$ & $30(15)$ & $-6(14)$ & $3(12)$ & $-16(12)$ \\
\hline$C(5 F)$ & $170(40)$ & $33(18)$ & $37(19)$ & $4(16)$ & $-60(20)$ & $-10(20)$ \\
\hline$C(6 F)$ & $40(13)$ & $44(14)$ & $51(15)$ & $-5(12)$ & $-8(11)$ & $-4(11)$ \\
\hline $\mathrm{C}(7 \mathrm{~F})$ & $150(20)$ & $100(20)$ & $80(20)$ & $-48(17)$ & $-40(16)$ & $35(17)$ \\
\hline $\mathrm{C}(8 \mathrm{~F})$ & $55(16)$ & $65(17)$ & $110(20)$ & $-41(15)$ & $9(14)$ & $-3(13)$ \\
\hline$S(7)$ & $62(4)$ & $10(3)$ & $27(3)$ & $0(3)$ & $-25(3)$ & $3(3)$ \\
\hline$C(1 G)$ & $29(8)$ & $20(8)$ & $18(8)$ & $6(7)$ & $-13(7)$ & $4(7)$ \\
\hline $\mathrm{C}(2 \mathrm{G})$ & $62(15)$ & $48(14)$ & $46(14)$ & $-35(12)$ & $-8(11)$ & $10(11)$ \\
\hline$C(3 G)$ & 21(10) & $28(11)$ & $32(12)$ & $-10(10)$ & $10(9)$ & $-10(8)$ \\
\hline $\mathrm{C}(4 \mathrm{G})$ & $42(13)$ & $65(16)$ & $60(16)$ & $-18(13)$ & $-23(12)$ & $-5(11)$ \\
\hline$C(5 G)$ & $20(11)$ & $92(18)$ & $78(18)$ & $-26(14)$ & $-12(12)$ & $11(12)$ \\
\hline$C(6 G)$ & $120(20)$ & $80(20)$ & $100(20)$ & $-31(15)$ & $-73(17)$ & $26(15)$ \\
\hline$C(7 G)$ & $75(16)$ & $46(15)$ & $81(18)$ & $-19(13)$ & $-40(14)$ & $12(12)$ \\
\hline $\mathrm{C}(8 \mathrm{G})$ & $60(15)$ & $50(15)$ & $40(14)$ & $9(12)$ & $-17(12)$ & $3(12)$ \\
\hline $\mathrm{S}(8)$ & $28(3)$ & $21(3)$ & $29(3)$ & $-16(3)$ & $-6(2)$ & $-2(2)$ \\
\hline $\mathrm{C}(1 \mathrm{H})$ & $33(13)$ & $52(17)$ & $100(30)$ & $-59(19)$ & $-39(16)$ & $22(12)$ \\
\hline $\mathrm{C}(2 \mathrm{H})$ & $31(12)$ & $16(11)$ & $21(12)$ & $1(10)$ & $-18(10)$ & $9(9)$ \\
\hline $\mathrm{C}(3 \mathrm{H})$ & $30(10)$ & $16(10)$ & $19(10)$ & $-2(8)$ & $-10(8)$ & $0(8)$ \\
\hline $\mathrm{C}(4 \mathrm{H})$ & 81(19) & $8(11)$ & $45(17)$ & $-3(11)$ & $-46(16)$ & $-2(11)$ \\
\hline $\mathrm{C}(5 \mathrm{H})$ & $64(19)$ & $44(18)$ & $60(20)$ & $-36(17)$ & $-13(16)$ & $-2(14)$ \\
\hline $\mathrm{C}(6 \mathrm{H})$ & $80(15)$ & $60(15)$ & $68(17)$ & $-22(13)$ & $-46(13)$ & $12(11)$ \\
\hline $\mathrm{C}(7 \mathrm{H})$ & $49(15)$ & $16(12)$ & $90(20)$ & $-21(14)$ & $-38(16)$ & $11(10)$ \\
\hline $\mathrm{C}(8 \mathrm{H})$ & $41(12)$ & $25(11)$ & $39(13)$ & $-20(10)$ & $-9(10)$ & $2(9)$ \\
\hline
\end{tabular}




\begin{tabular}{lllllll}
$\mathrm{S}(9)$ & $17(3)$ & $32(3)$ & $36(4)$ & $-17(3)$ & $-2(2)$ & $-4(2)$ \\
$\mathrm{C}(1 \mathrm{I})$ & $25(8)$ & $40(9)$ & $40(10)$ & $-16(7)$ & $-13(7)$ & $-9(7)$ \\
$\mathrm{C}(2 \mathrm{I})$ & $16(8)$ & $30(9)$ & $45(10)$ & $-10(7)$ & $-9(7)$ & $-14(6)$ \\
$\mathrm{C}(3 \mathrm{I})$ & $40(12)$ & $64(15)$ & $66(15)$ & $-33(12)$ & $-29(11)$ & $-13(10)$ \\
$\mathrm{C}(4 \mathrm{I})$ & $31(11)$ & $39(13)$ & $66(16)$ & $-29(12)$ & $-6(11)$ & $4(10)$ \\
$\mathrm{C}(5 \mathrm{I})$ & $10(9)$ & $61(14)$ & $54(14)$ & $-34(12)$ & $9(9)$ & $-6(9)$ \\
$\mathrm{C}(6 \mathrm{I})$ & $41(12)$ & $44(13)$ & $36(13)$ & $-16(11)$ & $-2(10)$ & $-9(10)$ \\
$\mathrm{C}(7 \mathrm{I})$ & $76(17)$ & $70(17)$ & $88(19)$ & $-43(14)$ & $-40(14)$ & $-3(13)$ \\
$\mathrm{C}(8 \mathrm{I})$ & $28(13)$ & $60(20)$ & $100(20)$ & $-64(19)$ & $-30(13)$ & $11(12)$ \\
$\mathrm{C}(1)$ & $120(30)$ & $120(30)$ & $150(40)$ & $-30(30)$ & $-90(30)$ & $-30(20)$ \\
$\mathrm{C}(2)$ & $29(11)$ & $63(15)$ & $44(14)$ & $-17(12)$ & $-10(10)$ & $-19(10)$ \\
$\mathrm{C}(3)$ & $61(19)$ & $60(20)$ & $60(20)$ & $24(17)$ & $-46(18)$ & $-36(16)$ \\
$\mathrm{C}(4)$ & $40(17)$ & $100(30)$ & $60(20)$ & $-30(20)$ & $-29(17)$ & $13(17)$ \\
$\mathrm{C}(5)$ & $33(14)$ & $60(20)$ & $45(19)$ & $26(16)$ & $-21(14)$ & $-5(13)$ \\
$\mathrm{C}(6)$ & $84(17)$ & $110(20)$ & $64(17)$ & $-29(14)$ & $-56(15)$ & $6(14)$ \\
$\mathrm{C}(7)$ & $39(12)$ & $60(15)$ & $32(13)$ & $-20(11)$ & $-7(10)$ & $-17(11)$ \\
& & & & & & \\
\hline & & & & & \\
& & & & & & \\
& & & & & & \\
\end{tabular}


Table S7. Hydrogen coordinates ( x 10 $0^{4}$ and isotropic displacement parameters $\left(\AA^{2} \times 10^{3}\right)$ for $\mathrm{Au}_{24} \mathrm{Cd}(\mathrm{PET})_{18}$.

\begin{tabular}{|c|c|c|c|}
\hline $\mathrm{H}(1 \mathrm{~A} 1)$ & -222 & 3840 & 10247 \\
\hline $\mathrm{H}(1 \mathrm{~A} 2)$ & -340 & 3052 & 11146 \\
\hline $\mathrm{H}(2 \mathrm{~A} 1)$ & 1129 & 3324 & 9489 \\
\hline $\mathrm{H}(2 \mathrm{~A} 2)$ & 1008 & 2519 & 10397 \\
\hline $\mathrm{H}(4 \mathrm{~A})$ & 1373 & 2648 & 11550 \\
\hline $\mathrm{H}(5 \mathrm{~A})$ & 2440 & 3532 & 11520 \\
\hline $\mathrm{H}(6 \mathrm{~A})$ & 2289 & 4982 & 10917 \\
\hline $\mathrm{H}(7 \mathrm{~A})$ & 1956 & 5562 & 9610 \\
\hline $\mathrm{H}(8 \mathrm{~A})$ & 972 & 4678 & 9603 \\
\hline $\mathrm{H}(1 \mathrm{~B} 1)$ & 1122 & -457 & 12599 \\
\hline $\mathrm{H}(1 \mathrm{~B} 2)$ & 2175 & -341 & 11929 \\
\hline $\mathrm{H}(2 \mathrm{~B} 1)$ & 1995 & -651 & 13400 \\
\hline $\mathrm{H}(2 \mathrm{~B} 2)$ & 2547 & 216 & 12712 \\
\hline $\mathrm{H}(4 \mathrm{~B})$ & 232 & -534 & 14080 \\
\hline $\mathrm{H}(5 \mathrm{~B})$ & -880 & 177 & 14863 \\
\hline $\mathrm{H}(6 \mathrm{~B})$ & -508 & 1468 & 14719 \\
\hline $\mathrm{H}(7 \mathrm{~B})$ & 976 & 2049 & 13791 \\
\hline $\mathrm{H}(8 \mathrm{~B})$ & 2089 & 1338 & 13007 \\
\hline $\mathrm{H}(1 \mathrm{C} 1)$ & 4107 & 388 & 6886 \\
\hline $\mathrm{H}(1 \mathrm{C} 2)$ & 3875 & 774 & 7609 \\
\hline $\mathrm{H}(2 \mathrm{C} 1)$ & 4975 & -82 & 8126 \\
\hline $\mathrm{H}(2 \mathrm{C} 2)$ & 5181 & -513 & 7450 \\
\hline $\mathrm{H}(4 \mathrm{C})$ & 5614 & 1334 & 7564 \\
\hline $\mathrm{H}(5 \mathrm{C})$ & 6572 & 2385 & 6490 \\
\hline $\mathrm{H}(6 \mathrm{C})$ & 7456 & 2308 & 5236 \\
\hline $\mathrm{H}(7 \mathrm{C})$ & 7291 & 1287 & 4835 \\
\hline
\end{tabular}




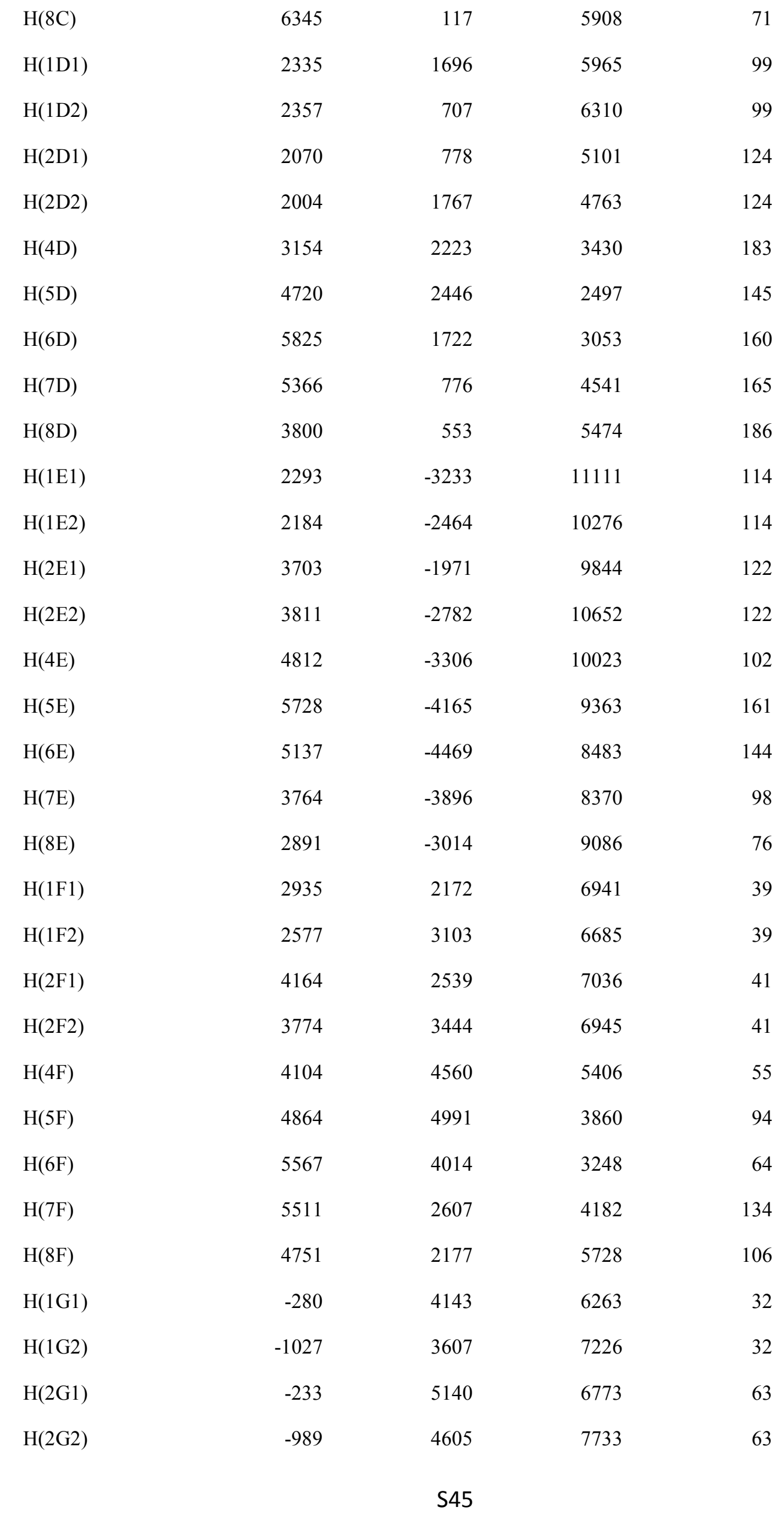




\begin{tabular}{|c|c|c|c|c|}
\hline $\mathrm{H}(4 \mathrm{G})$ & -2841 & 4647 & 7882 & 68 \\
\hline $\mathrm{H}(5 \mathrm{G})$ & -3630 & 5775 & 7232 & 84 \\
\hline $\mathrm{H}(6 \mathrm{G})$ & -3237 & 6362 & 5670 & 113 \\
\hline $\mathrm{H}(7 \mathrm{G})$ & -1647 & 6560 & 4955 & 81 \\
\hline $\mathrm{H}(8 \mathrm{G})$ & -532 & 5835 & 5501 & 74 \\
\hline $\mathrm{H}(1 \mathrm{H} 1)$ & -1759 & 2508 & 12151 & 57 \\
\hline $\mathrm{H}(1 \mathrm{H} 2)$ & -2578 & 2274 & 13119 & 57 \\
\hline $\mathrm{H}(2 \mathrm{H} 1)$ & -1518 & 2293 & 13746 & 29 \\
\hline $\mathrm{H}(2 \mathrm{H} 2)$ & -663 & 2448 & 12811 & 29 \\
\hline $\mathrm{H}(4 \mathrm{H})$ & -111 & 3875 & 11997 & 47 \\
\hline $\mathrm{H}(5 \mathrm{H})$ & -275 & 5273 & 11736 & 63 \\
\hline $\mathrm{H}(6 \mathrm{H})$ & -1761 & 5779 & 12224 & 78 \\
\hline $\mathrm{H}(7 \mathrm{H})$ & -3002 & 4884 & 12999 & 57 \\
\hline $\mathrm{H}(8 \mathrm{H})$ & -2850 & 3430 & 13538 & 43 \\
\hline $\mathrm{H}(1 \mathrm{I} 1)$ & 3368 & 918 & 10752 & 40 \\
\hline $\mathrm{H}(1 \mathrm{I} 2)$ & 4123 & 230 & 10869 & 40 \\
\hline $\mathrm{H}(2 \mathrm{I} 1)$ & 4717 & 1496 & 10557 & 38 \\
\hline $\mathrm{H}(2 \mathrm{I} 2)$ & 5308 & 1228 & 9744 & 38 \\
\hline $\mathrm{H}(4 \mathrm{I})$ & 3419 & 2586 & 10243 & 57 \\
\hline $\mathrm{H}(5 \mathrm{I})$ & 3225 & 3826 & 9192 & 54 \\
\hline $\mathrm{H}(6 \mathrm{I})$ & 4332 & 4388 & 7771 & 53 \\
\hline $\mathrm{H}(7 \mathrm{I})$ & 5629 & 3600 & 7252 & 84 \\
\hline $\mathrm{H}(8 \mathrm{I})$ & 5745 & 2293 & 8324 & 63 \\
\hline $\mathrm{H}(1 \mathrm{~A})$ & 7107 & 4564 & 5539 & 182 \\
\hline $\mathrm{H}(1 \mathrm{~B})$ & 7471 & 4530 & 4581 & 182 \\
\hline $\mathrm{H}(1 \mathrm{C})$ & 8083 & 4983 & 4790 & 182 \\
\hline $\mathrm{H}(3)$ & 8716 & 4189 & 3981 & 77 \\
\hline $\mathrm{H}(4)$ & 9751 & 3090 & 4066 & 79 \\
\hline $\mathrm{H}(5)$ & 9933 & 2176 & 5290 & 72 \\
\hline $\mathrm{H}(6)$ & 8869 & 2232 & 6653 & 92 \\
\hline $\mathrm{H}(7)$ & 7878 & 3324 & 6598 & 52 \\
\hline
\end{tabular}

